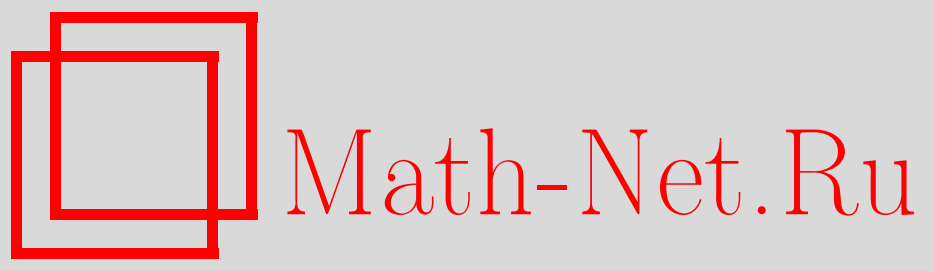

С. Б. Артамонов, А. Д. Миронов, А. Ю. Морозов, Иерархия дифференциалов и дополнительная градуировка полиномов узлов, ТМФ, 2014, том 179, номер 2, 147-188

DOI: https://doi.org/10.4213/tmf8625

Использование Общероссийского математического портала Math-Net.Ru подразумевает, что вы прочитали и согласны с пользовательским соглашением http://www.mathnet.ru/rus/agreement

Параметры загрузки:

IP: 54.166 .219 .16

26 апреля 2023 г., 11:42:11

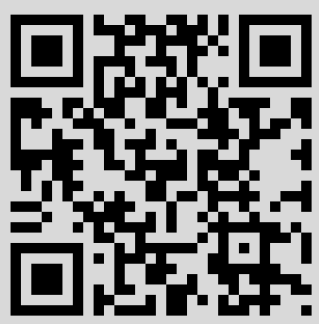


Том 179, № 2

май, 2014

(C) 2014 г. С. Б. Артамонов*, А. Д. Миронов*†, А. Ю. Морозов*

\title{
ИЕРАРХИЯ ДИФФЕРЕНЦИАЛОВ И ДОПОЛНИТЕЛЬНАЯ ГРАДУИРОВКА ПОЛИНОМОВ УЗЛОВ
}

\begin{abstract}
Раскрашенные полиномы узлов обладают специальным $Z$-разложением в определенные комбинации дифференциалов, которые зависят от представления. Коэффициенты разложения являются функциями трех переменных $A, q, t$ и могут быть рассмотрены как новые выделенные координаты в пространстве полиномов узлов, аналогичные коэффициентам альтернативного разложения по характерам. Эти новые переменные разлагаются особенно просто, когда представление погружено в произведение фундаментальных представлений. Недавно предложенная четвертая градуировка, по-видимому, является простым переопределением этих новых координат, элегантным, но никоим образом не выделенным. Если это так, то она не дает никаких новых независимых инвариантов узлов, но вместо этого может быть рассмотрена как еще одно свидетельство в пользу существования скрытой структуры иерархии дифференциалов $(Z$-разложения), стоящей за полиномами узлов.
\end{abstract}

Ключевые слова: теория Черна-Саймонса, цветные инварианты узлов, суперполиномы.

DOI: $10.4213 / \operatorname{tmf} 8625$

\section{1. ВВЕДЕНИЕ}

Полиномы узлов [1] являются наблюдаемыми (средними от вильсоновских петель) в одной из простейших моделей Янга-Миллса - теории Черна-Саймонса [2], [3] в односвязном трехмерном евклидовом пространстве-времени $M_{3}=\mathbb{R}^{3}$ или $M_{3}=S^{3}$ (в более сложных пространствах они оказываются даже более интересными неполиномиальными функциями). Изучение этих функций и богатого набора соотношений между ними очень важно для понимания общей структуры гравитационных моделей Янга-Миллса и более общих струнных моделей. Поскольку теория топологическая,

* Институт теоретической и экспериментальной физики, Москва, Россия.

E-mail: artamonov@itep.ru,mironov@itep.ru,morozov@itep.ru

${ }^{\dagger}$ Физический институт им. П. Н. Лебедева РАН, Москва, Россия. E-mail: mironov@lpi.ru 
средние от вильсоновских петель ${ }^{1)}$

$$
H_{R}^{\mathcal{K}} \sim\left\langle\operatorname{Tr}_{R} \operatorname{Pexp} \oint_{\mathcal{K}} \mathcal{A}\right\rangle
$$

являются относительно простыми - в действительности они зависят не от геометрии линии $\mathcal{K} \subset M_{3}$, а лишь от ее топологии, т. е. $\mathcal{K}$ можно рассмотреть как узел или зацепление (если он состоит из нескольких несвязанных линий). Среднее также зависит от представления $R$, от константы связи $q=e^{2 \pi i /(k+N)}$ и от калибровочной группы, в качестве которой мы выбираем $G=S U(N)$; таким образом, неприводимые представления $R$ будут конечномерными и будут отвечать диаграммам Юнга (обобщение на другие алгебры Ли, компактные и некомпактные, можно провести непосредственно). Как уже отмечалось, для односвязного пространства $M_{3}$ среднее $H_{R}$ оказывается полиномом (который называется полиномом ХОМФЛИ или ХОМФЛИ-ПТ ${ }^{2)}$ ) от непертурбативных переменных $q$ и $A=q^{N}$, где $\ln A$ - константа связи 'т Хоофта, которая остается конечной в планарном пределе теории Янга-Миллса.

Среднее $H_{R}$ - это не только полином, все его коэффициенты оказываются целыми, что подразумевает лежащую за этим дополнительную гомологическую структуру. Модули этих целых коэффициентов рассматриваются как нечто вроде (БПС) состояний некоторой скрытой топологической теории [4]. Действительно, $H_{R}$ может быть представлен как некоторый полином Эйлера комплекса Хованова-Рожанского [5], [6], который описывает флипы между разными разрешениями диаграммы узла и индуцирован операторами разрезания и склейки (см. недавний обзор в работе [7]). Соответствующий полином Пуанкаре имеет все положительные коэффициенты, но зависит от одного дополнительного параметра (градуировки) T, а первоначальный полином получается из этого "суперполинома" [8] при $T=-1$ :

$$
H_{R}^{\mathcal{K}}(A \mid q)=\left.P_{R}^{\mathcal{K}}(A \mid q, T)\right|_{T=-1} .
$$

Для полинома ХОМФЛИ $H_{R}$ в настоящее время существует очень эффективный способ его вычисления через сумму по путям на дереве представлений [9]. Этот способ основан на следующем:

- представлении полиномов узлов в терминах квантовых $\mathcal{R}$-матриц [10] в темпоральной калибровке $A_{0}=0$ в теории Черна-Саймонса (где пропагатор становится ультралокальным, он имеет вид $\left.\delta^{(2)}(\vec{x}) \operatorname{sign}(t)\right)$;

- представлении диаграммы узла в виде косы (двумерная проекция узла $\mathcal{K}$, которая естественно возникает в темпоральной калибровке);

- разложении по характерам [11], [12] (см. также работы [13] и [14]), которое сводит рассмотрение к много более простым $\widehat{\mathcal{R}}$-матрицам, действующим в пространстве сплетающих операторов.

1) В настоящей статье мы рассматриваем редуцированные полиномы узлов, деленные на полином, отвечающий окружности, отсюда знак вместо равенства.

${ }^{2)}$ По фамилиям Hoste, Ocneanu, Millet, Freyd, Lickorish, Yetter, Przytycki, Traczyk, см. статьи [1]. 
Этот метод обобщает, концептуально и технически, хорошо известный подход, основанный на скейн-соотношениях в фундаментальном представлении (в котором $\widehat{\mathcal{R}}$-матрица имеет в точности два собственных значения и удовлетворяет уравнению $(\mathcal{R}-q)(q \mathcal{R}+1)=0)$ и на использовании каблирования, сводящего вычисление раскрашенных полиномов узлов к вычислению полиномов в фундаментальном представлении для каблированных узлов (в современных терминах, достаточно ограничить все пути в дереве представлений на пути, проходящие через вершину $R$ ). Метод технически очень эффективен и позволяет вычислять раскрашенные полиномы ХОМФЛИ в весьма сложных примерах. Однако все еще неясно, как этот метод обобщается на суперполиномы.

До недавнего времени мы знали несколько примеров суперполиномов из двух источников: из "ручного" анализа частных случаев, проведенного в работе [8] и последующих статьях [13], [15], [16], и из более систематического метода эволюции [11], [17], который, однако, требует использования нескольких простых частных случаев в качестве начальных данных. Наиболее впечатляющее применение метода эволюции - торические узлы. В этом случае на основе первоначального анализа [11], [13] и специфического "торического" подхода [18] удалось предложить полное решение в терминах двойных аффинных алгебр Гекке [14], [19]. Однако остается неясным, до какой степени метод эволюции систематически применим при этом подходе, и, что более существенно, ответы для предполагаемых суперполиномов не всегда положительны. Это может означать, что они все-таки скорее описывают некоторую нетривиальную деформацию полинома Эйлера, нежели полином Пуанкаре. Если это так, то подразумевается существование новых нетривиальных деформаций (дополнительные градуировки), по крайней мере для торических узлов.

$\mathrm{K}$ счастью, сегодня имеется другой мощный метод систематического вычисления суперполиномов - метод $Z$-разложения [20], представляющий собой далекое обобщение оригинального рассмотрения в работе [8]. Он все еще развит недостаточно, но уже позволил вычислить раскрашенные суперполиномы во всех (анти)симметрических представлениях для серий рациональных узлов (твистованных узлов [21] и торических узлов [22], в этих случаях метод замечательно согласован с методом эволюции, позволяющим получить даже больше результатов [17]); также $Z$-разложение понемногу применяется к менее тривиальным представлениям [23], [24]. Что еще более важно, этот метод дает совсем другое описание полиномов узлов и проливает новый свет на проблему дополнительных градуировок. В частности, недавно предложенная четвертая градуировка [24], по-видимому, является одной из многих возможных новых градуировок. Этот подход помогает понять, что тем не менее все градуировки не столь существенны, как первоначальные три переменные $A, q, T$. В то же время новые примеры суперполиномов, найденные в работе [24], позволяют продвинуться в понимании $Z$-разложений. Возможно, более адекватным названием было бы “иерархия дифференциалов", что отсылает нас к “дифференциалам" Хованова и Дринфельда-Гукова-Расмуссена (ДГР) [5], [8]. Что касается буквы $Z$ в названии, то она напоминает нам о важных случаях, когда дифференциалы входят парами, которые в работе [20] названы $Z$-факторами (хотя это буквально верно только для прямоугольных диаграмм Юнга). Далее мы используем оба термина как равноправные. 


\section{2. ИДЕЯ Z-РАЗЛОЖЕНИЯ}

Современный подход к корреляторам в квантовой теории поля в общем и к средним от вильсоновских петель в теории Черна-Саймонса в частности состоит в том, что мы сопоставляем любому узлу $\mathcal{K}$ и представлению $R$ следующий элемент бесконечномерного векторного пространства:

$$
X_{R}^{\mathcal{K}}=\sum_{I} x_{R}^{\mathcal{K}}[I] e[I]
$$

где $\left\{x_{R}^{\mathcal{K}}[I]\right\}$ - набор коэффициентов разложения по фиксированному базису $\{e[I]\}$, который не зависит от узла и представления. Тогда корреляционная функция может рассматриваться как спаривание $\left\langle\mathrm{vac} \mid X_{R}^{\mathcal{K}}\right\rangle$ этого элемента и точки в дуальном пространстве, называемой вакуумом. В работах [11], [12], [25] элементы, подобные (2.1), которые соответствуют средним от вильсоновских петель в теории Черна-Саймонса и называются расширенные полиномы узлов (или полиномы узлов вне массовой оболочки), были введены в пространстве симметрических функций с базисами характеров, функций Шура и полиномов Макдональда от (бесконечного числа) временны́х переменных $\left\{p_{k}\right\}$. Спаривание в этом случае эквивалентно редукции базиса на топологический локус

$$
p_{k}^{*}=\frac{A^{k}-A^{-k}}{t^{k}-t^{-k}}, \quad M_{R}\left(p^{*}\right)=M_{R}^{*}
$$

(при $A=t^{N}$ и $t=q$ данные $M_{R}^{*}$ являются в точности обычными квантовыми размерностями), при этом объекты вне массовой поверхности $X_{R}$ редуцируются к полиномам ХОМФЛИ узла $H_{R}$ (и суперполиномам $P_{R}$ ) на массовой поверхности. Здесь $q$ и $t$ - параметры полиномов Макдональда. Мы в основном используем переменную $t=-q / T$ вместо $T$. Как правило, существуют разные разложения вне массовой оболочки по базису характеров, ассоциированному с разными представлениями одного и того же узла в виде косы (т. е. полиномы узлов вне массовой поверхности более не являются топологическим инвариантом, но взамен, по крайней мере для торических узлов, приводят к $\tau$-функциям иерархии КП и представляются зависящей от временнь́х переменных матричной моделью). При этом полиномы вне массовой поверхности $X_{R}^{\mathcal{K}}$ различаются, но на массовой поверхности все они совпадают и не зависят от выбора косы.

В настоящей статье мы развиваем подход, основанный на альтернативном предложении [17], [20] разлагать полиномы узлов вне массовой оболочки не по базису характеров $M_{Q}^{*}(A \mid q, t)$, как в работах [9], [11]-[14], а по некоторому совершенно иному базису, составленному из дифференциалов

$$
d[I]=d_{j_{1} \ldots j_{k}}^{i_{1} \ldots i_{k}},
$$

которые на массовой поверхности редуцируются к "мультидифференциалам":

$$
d[I] \rightarrow \prod_{a=1}^{k} D_{j_{a}}^{i_{a}} \equiv \prod_{a=1}^{k}\left\{\frac{A q^{i_{a}}}{t^{j_{a}}}\right\}, \quad\{x\} \equiv x-x^{-1} .
$$

Они являются просто полиномами (Лорана), слово “дифференциалы” связано с их ролью в теории гомологий Хованова. Причина, почему эти величины изначально 
важны в теории узлов, состоит в том, что теория представлений для конкретных значений $N$ отвечает обращению в ноль элементарных дифференциалов, подобных $\left\{A q^{N}\right\}$ и $\left\{A / t^{N}\right\}$, и полиномы узлов строятся так, чтобы эти свойства выполнялись (см. детальное обсуждение в работах [8], [20], [23]).

Таким образом, мы имеем два примера элемента (2.1):

$$
x_{R}^{\mathcal{K}}[I]=g_{R}^{\mathcal{K}}[I], \quad e[I]=d[I],
$$

если $I$ является набором целых чисел, и

$$
x_{R}^{\mathcal{K}}[I]=c_{R}^{\mathcal{K}}[I], \quad e[I]=M_{I},
$$

если $I$ - диаграмма Юнга. Полиномы узлов в базисе мультидифференциалов задаются коэффициентами разложения $g^{\mathcal{K}}$, которые играют ту же роль, что и $c^{\mathcal{K}}$ в разложении по характерам. Заметим, что топологическая инвариантность в разложении по дифференциалам фиксирует его нормировку так, что разложение начинается с единицы. В обоих случаях - разложения по характерам или разложения по дифференциалам - базисы $\left(M_{Q}^{*}\right.$ или $\left.D[I]\right)$ универсальны, т. е. одинаковы для всех узлов. От узла $\mathcal{K}$ зависят коэффициенты разложения $\left(c_{R}^{\mathcal{K}}[I]\right.$ и $\left.g_{R}[I]\right)$.

Ключевым преимуществом разложения по дифференциалам является то, что оно контролирует зависимость коэффициентов от представления $R$ и от параметров $q$ и $t$. На самом деле в обоих случаях это нечто вроде двойной деформации первичного соотношения для специальных полиномов $\sigma^{\mathcal{K}}$ [11] (полиномов узлов при $t=q=1$ ), когда все дифференциалы сводятся просто к степени $\{A\}:$ при $t=q=1$ мы имеем

$$
P_{R}^{\mathcal{K}}=\left(P_{\square}^{\mathcal{K}}\right)^{|R|}=\left(\sigma^{\mathcal{K}}\right)^{|R|},
$$

следовательно,

$$
\begin{aligned}
\sum_{Q \vdash m|R|} c_{R Q}^{\mathcal{K}} M_{Q}^{*} & =\left(\sum_{Q \vdash m} c_{\square Q}^{\mathcal{K}} M_{Q}^{*}\right)^{|R|}, \\
1+\sum_{I} g_{R}^{\mathcal{K}}[I]\{A\}^{2 I} & =\left(1+\sum_{I} g_{\square}^{\mathcal{K}}[I]\{A\}^{2 I}\right)^{|R|} .
\end{aligned}
$$

Однако в случае разложения по дифференциалам происходит чудо: деформация практически непосредственная. Этот факт был ясно продемонстрирован в работе [20] для узла-“восьмерки": стартуя со специального полинома $\sigma^{4_{1}}=1+\{A\}^{2}$, можно определить процедуру, в результате которой получаются не только полиномы ХОМФЛИ, но и суперполиномы во всех симметрических и антисимметрических представлениях. В работе [23] указанная процедура была обобщена на представление $R=[21]$, а также обсуждались некоторые указания на то, что она может быть расширена далее на произвольные представления. Более того, та же процедура одновременно дает раскрашенные суперполиномы для трилистника, если начинать с $Z$-разложения его специального полинома $\sigma^{3_{1}}=1-A^{2}\{A\}^{2}$. Задачей настоящей статьи является сделать утверждение о том, что разложение по дифференциалам открывает ясный путь для контроля зависимости полиномов узлов от представления, более общим - распространить его на произвольные узлы. 
А именно, кажется все более и более правдоподобным, что при разложении полиномов узлов по базису $D[I]$, т. е. при использовании коэффициентов этого разложения как новых координат в пространстве узлов, независимыми координатами являются в действительности коэффициенты этого разложения для специальных полиномов. Критичным является, конечно, тот факт, что имеет значение не суперполином сам по себе, но его соответствующее $Z$-разложение, т. е. некоторая дополнительная структура, зная которую, можно понять многое (возможно, всё) об узле. Разница может показаться неясной в приведенном выше примере узла $4_{1}$, она несколько лучше видна для трилистника с $\sigma^{3_{1}}=-A^{4}+2 A^{2}=1-A^{2}\{A\}^{2}$ и становится вполне впечатляющей для более сложных двухнитевых узлов. Например,

$$
\sigma^{[2,7]}=4 A^{6}-3 A^{8}=1-\left(3 A^{2}+2 A^{6}+A^{10}\right)\{A\}^{2}+\left(3 A^{4}+2 A^{8}\right)\{A\}^{4}-A^{6}\{A\}^{6}
$$

отвечает четырехкомпонентному вектору

$$
\left.g_{\square}^{[2,7]}\right|_{q=t=1}=\left[1,-\left(3 A^{2}+2 A^{6}+A^{10}\right), 3 A^{4}+2 A^{8},-A^{6}\right]
$$

(совсем не то же, что простое двучленное выражение для $\sigma^{[2,7]}$, который потенциально мог бы однозначно задавать узел. Семикомпонентный вектор

$$
\begin{aligned}
g_{[2]}^{[2,7]} & =g_{\square}^{[2,7]} \otimes g_{\square}^{[2,7]}= \\
& =\left[1,-2\left(3 A^{2}+2 A^{6}+A^{10}\right), 2\left(3 A^{4}+2 A^{8}\right)+\left(3 A^{2}+A^{6}+A^{10}\right)^{2}, \ldots, A^{12}\right]
\end{aligned}
$$

может служить стартовой точкой для двух различных и определенных алгоритмически $(q, t)$-деформаций. Эти деформации дадут два суперполинома $P^{[2,7]}$ в представлениях $R=[2]$ и $R=[11]$. В действительности ситуация даже еще сложнее: как объясняется в работе [20], с точки зрения $Z$-разложения вектор, подобный (2.5), имеет внутреннюю структуру, о которой свидетельствуют отличные от единицы коэффициенты, и истинно адекватные координаты содержат даже больше компонент (см. примеры в разделах 3,4$)$.

Иными словами, можно говорить о растущей очевидности того, что коэффициенты $g_{\square}^{\mathcal{K}}[I]$ содержат значительно больше информации об узле $\mathcal{K}$, чем $c_{\square}^{\mathcal{K}}$. Следовательно, в этой параметризации деформацию (2.4) можно понять намного лучше. Можно даже думать, что она полностью алгоритмическая, тогда набор функций $g_{\square}^{\mathcal{K}}[I](A \mid q, t)$ с $R=\square$, т. е. ассоциированных только с фундаментальным представлением (возможно, даже набор $g_{\square}^{\mathcal{K}}[I](A \mid q)$ при $q=t$, т. е. эти переменные для набора полиномов ХОМФЛИ) может обеспечить полную информацию о полиномах узлов ${ }^{3)}$. Это утверждение (которое мы иллюстрируем ниже многочисленными примерами) звучит странно, и может показаться, что оно противоречит тому, что раскрашенные суперполиномы содержат больше информации, чем фундаментальные полиномы ХОМФЛИ. Секрет, конечно, в том, что набор $g_{\square}^{\mathcal{K}}[I]$ содержит намного больше информации, чем просто $H_{\square}^{\mathcal{K}}$. Последний получается из первого на массовой поверхности, т. е. из (2.1), когда $d[I]$ - не просто свободные параметры, но выражения

3)Эта гипотеза выглядит очень вероятной для полиномов ХОМФЛИ и для суперполиномов в (анти)симметрических представлениях, в то время как ее статус для более сложных представлений остается неясным. 
из формулы (2.3). После этого происходит множество сокращений, и выражение кардинально упрощается.

Мы увидим в п. 3.4, что двухнитевые торические фундаментальные полиномы ХОМФЛИ, которые представляют собой просто квадратичные полиномы по $A$ (с точностью до нормировки), описываются огромными наборами ненулевых величин $g_{\square}[I]$, являющихся полиномами высших степеней по $A$. Другой стороной этой медали является то, что совсем не просто выделить $g$-переменные, даже если полиномы ХОМФЛИ известны: либо нужно знать их в достаточно большом количестве представлений, либо иметь глубокое понимание скрытой структуры разложения по дифференциалам. Как бы то ни было, мы предполагаем, что такая структура существует, и что $g$-переменные задают замечательный набор координат в пространстве узлов. Эти переменные могут выглядеть излишне усложненными в частных примерах, но зато они адекватно отражают структуру соотношений между различными полиномами узлов. В частности, четвертая градуировка, предложенная в работе [24], может интерпретироваться как нечто подобное преобразованию $g$-переменных (и имеется множество других переменных того же типа, возможно, менее элегантных, но равно допустимых): если это верно, данная дополнительная градуировка сильно отличается от $A, q, t$.

Интуитивное представление состоит в том, что с каждым представлением $R$ ассоциируется пара операций, которые, действуя совместно, превращают $g_{\square}$-переменные в $g_{R}$-переменные:

$$
g_{R}\left[I_{R}\right]=\left(g_{\square}[I]\right)^{\circ|R|} .
$$

Одна из этих операций $\left(I_{R}\right)$ преобразует набор $I$, в то время как другая $\left(\circ_{R}\right)$ определяет подходящую свертку коэффициентных функций. Тем не менее этого понимания уже достаточно для того, чтобы сделать изучение иерархии дифференциалов [20] и ее сравнение с разложением по характерам, суммой по путям и методом эволюции вполне важным и интересным.

Мы начинаем наше рассмотрение с простейшего примера узла-“восьмерки" $4_{1}$ в симметрических представлениях, где функции $g[I]$ по сути тривиальны. Следовательно остается только операция $I_{R}$. После этого пример трилистника $3_{1}$ демонстрирует, что операция о также может быть довольно простой. Однако для более общих твистованных узлов уже имеются проблемы, и это требует дальнейшего изучения.

\section{3. БАЗОВЫЕ ПРИМЕРЫ ИЕРАРХИИ ДИФФЕРЕНЦИАЛОВ: (АНТИ)СИММЕТРИЧЕСКИЕ ПРЕДСТАВЛЕНИЯ}

3.1. Узел-“восьмерка" 4 . Материал данного пункта можно найти в рабо$\operatorname{Tax}[20],[23]$.

Несмотря на то что представление в виде косы для узла-“восьмерки” включает по меньшей мере три нити, этот узел, простейший из полностью симметричных узлов, оказывается также простейшим с точки зрения раскрашенных полиномов узлов и, в особенности, иерархии дифференциалов. В частности, ответ для полинома узла 
в фундаментальном представлении совершенно тривиален:

$$
H_{\square}^{4_{1}}(A \mid q)=1+\{A q\}\left\{\frac{A}{t}\right\} \quad \Longrightarrow \quad P_{\square}^{4_{1}}(A \mid q, t)=1+\{A q\}\left\{\frac{A}{t}\right\}=1+D_{01}^{10} .
$$

Эта формула отражает первое базисное свойство иерархии дифференциалов: выражения для полиномов ХОМФЛИ прямо поднимаются до выражений для суперполиномов, как только они записаны в терминах мультидифференциалов (2.3). В этом случае единственный неисчезающий параметр - это $\mathcal{K}=4_{1}$ : мы имеем $g_{\square}^{4_{1}}\left[\begin{array}{l}01 \\ 10\end{array}\right]=1$.

Вторая особенность отражена в архетипичных формулах [20] для раскрашенных полиномов узлов в симметрических представлениях:

$$
P_{[r]}^{4_{1}}(A \mid q, t)=1+\sum_{j=1}^{r} \frac{[r]_{q} !}{[j]_{q} ![r-j]_{q} !} \prod_{i=0}^{j-1} D_{0}^{r+i} D_{1}^{i}
$$

Здесь квантовые числа определены как

$$
[x]_{q}=\frac{q^{x}-q^{-x}}{q-q^{-1}}=[x]_{1 / q} .
$$

В антисимметрическом представлении полиномы

$$
P_{\left[1^{r}\right]}^{4_{1}}(A \mid q, t)=1+\sum_{j=1}^{r} \frac{[r]_{t} !}{[j]_{t} ![r-j]_{t} !} \prod_{i=0}^{j-1} D_{r+i}^{0} D_{i}^{1}
$$

получаются из (3.1) “зеркальным" отображением [11], [16]

$$
q \longleftrightarrow-t^{-1}, \quad \text { т. е. } \quad D_{j}^{i} \longleftrightarrow D_{i}^{j} \text {. }
$$

Может показаться, что полиномы в антисимметрическом представлении не дают ничего нового. Однако знание обеих формул важно для изучения общих представлений. Эти формулы можно переписать множеством способов, один из наиболее важных при этом превращает $q$-биномиальные коэффициенты в расширенный набор дифференциалов [20]. Это объясняет, в каком смысле такие комбинаторные факторы могут интерпретироваться как описывающие набор $I(R)$ в (2.2). K примеру, правильный способ смотреть на первый член в сумме (3.1) - это заменить его комбинацией с единичными коэффициентами:

$$
[r]_{q} D_{1}^{r}=\sum_{i=0}^{r} D_{1}^{2 i}
$$

например

$$
[2]_{q}\left\{A q^{2}\right\}\left\{\frac{A}{t}\right\}=\left\{A q^{3}\right\}\left\{\frac{A}{t}\right\}+\{A q\}\left\{\frac{A}{t}\right\} .
$$

Подобным образом можно обращаться со всеми остальными биномиальными коэффициентами. Популярны также замены $q$-факториалов на $q$-символы Похгамера и на другие наборы переменных $(A, q, t)$.

Из этих формул можно извлечь два непосредственных урока. 
Во-первых, при умножении представлений коэффициенты преобразуются простым образом. Набор дифференциалов, дающих вклад в (3.1) и (3.2), ограничен, так что эти формулы могут быть переписаны как

$$
\begin{aligned}
P_{[r]}^{4_{1}}(A \mid q, t) & =1+\sum_{j=1}^{r} g_{r \mid j} \prod_{i=0}^{j-1} D_{0}^{r+j} D_{1}^{j}, & g_{r \mid j} & =\frac{[r]_{q} !}{[j]_{q} ![r-j]_{q} !}, \\
P_{\left[1^{r}\right]}^{4_{1}}(A \mid q, t) & =1+\sum_{j=1}^{r} \bar{g}_{r \mid j} \prod_{i=0}^{j-1} D_{r+j}^{0} D_{j}^{1}, & \bar{g}_{r \mid j} & =\frac{[r]_{t} !}{[j]_{t} ![r-j]_{t} !} .
\end{aligned}
$$

Если рассмотреть производящие функции

$$
\pi_{[r]}^{4_{1}}(z)=1+\sum_{j=0}^{r} g_{r \mid j} z^{2 j}, \quad \bar{\pi}_{\left[1^{r}\right]}^{4_{1}}(z)=1+\sum_{j=0}^{r} \bar{g}_{r \mid j} z^{2 j}
$$

Tо

$$
\pi_{[r]}^{4_{1}}(z)=\left[\pi_{\square}^{4_{1}}(z)\right]_{q}^{r}, \quad \bar{\pi}_{\left[1^{r}\right]}^{4_{1}}(z)=\left[\bar{\pi}_{\square}^{4_{1}}(z)\right]_{t}^{r},
$$

где квантовая степень, или q-символ Похгамера определяется как

$$
[1+x]_{q}^{r}=1+\sum_{j=1}^{r} g_{r \mid j} x^{j}=\prod_{j=1}^{r}\left(1+q^{2 j-r-1} x\right) .
$$

Эти формулы ясно демонстрируют, что для различных представлений (представлений $[r]$ и $\left[1^{r}\right]$ в данном случае) операции $I_{R}$ и $\circ_{R}$ различны. В то же время разница контролируется структурой диаграммы Юнга весьма простым и интуитивно понятным способом.

Во-вторых, по модулю тривиальных комбинаторных факторов коэффициенты разложения (3.1) не зависят от представления: если положить

$$
g_{r \mid j}=\frac{[r]_{q} !}{[j]_{q} ![r-j]_{q} !} G_{j}
$$

то $G$ перестает зависеть от $r$; более того, для любого $j$

$$
G_{j}^{4_{1}}=1
$$

для других узлов это полиномы Лорана от $A, q$ и $t$, причем параметр $T=-q / t$ легко восстанавливается из случая ХОМФЛИ, когда $T=-1$.

Другой путь закодировать эти соотношения состоит в том, чтобы написать разностное уравнение $[20]^{4)}$

$$
P_{[r+1]}^{4_{1}}(A)-P_{[r]}^{4_{1}}(A)=\left\{A q^{2 r+1}\right\}\left\{\frac{A}{t}\right\} P_{[r]}^{4_{1}}(q A) .
$$

\footnotetext{
4) Связь разностного уравнения с уравнениями для квантовых А-полиномов [21], [26] до сих пор неясна, хотя эти полиномы замечательны с точки зрения установления связей с уравнениями Бакстера, ассоциированными с пятимерными калибровочными теориями [27].
} 
Сдвиг $A \rightarrow q A$ в правой части отвечает за перемешивание набора дифференциалов, ассоциированных с набором соотношений

$$
\begin{aligned}
& \left([r+1]_{q}\left\{A q^{r+1}\right\}-\underline{[r]_{q}\left\{A q^{r}\right\}}\left\{A q^{2 r+1}\right\}\right) \cdot \underline{\left\{\frac{A}{t}\right\}} \cdot \underline{\underline{1}}=\left\{A q^{2 r+1}\right\} \cdot \underline{\left\{\frac{A}{t}\right\}} \cdot \underline{\underline{1}}, \\
& \left([r+1]_{q}\left\{A q^{r+2}\right\}-\underline{[r-1]_{q}\left\{A q^{r}\right\}}\right) \cdot \underline{\left(\frac{A}{t}\right\}} \cdot \underline{\underline{\left([r]_{q}\left\{A q^{r+1}\right\}\left\{\frac{A q}{t}\right\}\right)}}= \\
& =[2]_{q}\left\{A q^{2 r+1}\right\} \cdot \underline{\left\{\frac{A}{t}\right\}} \cdot \underline{\underline{\left([r]_{q}\left\{A q^{r+1}\right\}\left\{\frac{A q}{t}\right\}\right)}}, \\
& \left([r+1]_{q}\left\{A q^{r+3}\right\}-\underline{[r-2]_{q}\left\{A q^{r}\right\}}\right) \cdot \underline{\left\{\frac{A}{t}\right\}} \cdot \underline{\underline{[r]_{q}[r-1]_{q}\left\{A q^{r+2}\right\}\left\{A q^{r+1}\right\}\left\{\frac{A q^{2}}{t}\right\}\left\{\frac{A q}{t}\right\}}}= \\
& =[3]_{q}[2]_{q}\left\{A q^{2 r+1}\right\} \cdot\left\{\frac{A}{t}\right\} \cdot[r]_{q}[r-1]_{q}\left\{A q^{r+2}\right\}\left\{A q^{r+1}\right\}\left\{\frac{A q^{2}}{t}\right\}\left\{\frac{A q}{t}\right\}
\end{aligned}
$$

и т. д., где дважды подчеркнутые дифференциалы отличаются от однократно подчеркнутых в предыдущей строке сдвигом $A \rightarrow q A$.

ЗАмЕчАниЕ 1. Вышеприведенные примеры объясняют, чего мы хотим от $Z$-разложения, т. е. обеспечивают что-то вроде описания, по меньшей мере концептуально. В рамках этого подхода узлы описываются (бесконечным) набором $G$-параметров, которые не зависят от представления, а $Z$-разложение обеспечивает процедуру реконструкции произвольного раскрашенного суперполинома, если мы знаем эти параметры.

Последующие примеры даны, чтобы продемонстрировать, что такая процедура реконструкции действительно может существовать. Также они показывают, что четвертая градуировка [24] действует как тривиальное перемасштабирование в пространстве $G$-параметров (но, поскольку мультидифференциалы при этом не преобразуются, полиномы узлов, которые получаются в результате, могут меняться, в большинстве случаев это и происходит, см. примеры в разделе 5).

3.2. Трилистник. Наш следующий пример - это трилистник $3_{1}$, который является также простейшим торическим узлом. Данный пример поможет нам проиллюстрировать несколько важных вопросов.

В то время как набор подходящих дифференциалов в (анти)симметрических представлениях все еще может быть описан индексами $r \mid j$ с $j=1, \ldots, r$ (это характерная черта всех твистованных узлов), уже для трилистника не зависящие от представления коэффициенты $G_{j}$ начинают зависеть от $A, q, t$ :

$$
G_{j}^{3_{1}}=(-1)^{j} A^{2 j} q^{j(2 j-1)} t^{j(2 j-3)}=\left(-\frac{A^{2} q}{t}\right)^{j} q^{2 j(j-1)} .
$$

Вторая версия этой формулы демонстрирует, как полином ХОМФЛИ поднимается до суперполинома: нужно ввести параметр $t \neq q$ в дифференциалы, как в (2.3), 
а в коэффициентах нужно сделать замену

$$
A^{2} \rightarrow \frac{A^{2} q}{t}, \quad q^{2} \rightarrow q^{2},
$$

т. е. $q$-переменные остаются неизменными. Ожидается, что этот рецепт будет работать для всех твистованных узлов.

Возможно, наиболее важный пример трилистника позволяет нам проиллюстрировать разницу между разложениями по характерам и по дифференциалам. В этом примере она еще не столь существенна (как для более сложных торических узлов), но уже вполне заметна.

Как уже упоминалось, трилистник - это торический узел $[2,3]=[3,2]$, таким образом, разложение по характерам дается непосредственной $t$-деформацией [11] (напоминаем, что наши суперполиномы редуцированные) формулы Россо-Джонса [28]. В фундаментальном представлении имеем

$$
P_{\square}^{[2,3]}=A^{3}\left(t^{-3} \frac{\{A q\}}{\{q t\}}-q^{3} \frac{\{A / t\}}{\{q t\}}\right) .
$$

В то же время $Z$-разложение этой величины выглядит совершенно по-другому: с учетом формулы (3.9)

$$
P_{\square}^{3_{1}}=1-\frac{A^{2} q}{t}\{A q\}\left\{\frac{A}{t}\right\}=1-\frac{A^{2} q}{t} D_{01}^{10}, \quad H_{\square}^{3_{1}}=1-A^{2}\{A q\}\left\{\frac{A}{t}\right\} .
$$

Заметим, что выражение для той же величины в неструктурированном виде существенно отличается от обеих формул:

$$
P_{\square}^{\text {trefoil }}=-\frac{q^{2}}{t^{2}} A^{4}+A^{2}\left(q^{2}+t^{-2}\right), \quad H_{\square}^{\text {trefoil }}=-A^{4}+A^{2}\left(q^{2}+q^{-2}\right) .
$$

Конечно, все три формулы дают один и тот же результат, $P^{3_{1}}=P^{[2,3]}=P^{\text {trefoil }}$, но их смысл неодинаков, они принадлежат к разным классам, которые имеют совершенно разную структуру и смысл. $\mathrm{K}$ примеру, $P^{[2,3]}$ замечательно подходит для введения полиномов узлов вне массовой оболочки в базисе характеров [12]. Наоборот, $P^{3_{1}}$ лучше всего годится для продолжения на другие представления: как мы объясняли, цель $Z$-разложения - обеспечить аналог свойства факторизации (2.4), которое может быть поднято на случай $t \neq q \neq 1$. В данном частном примере это действительно можно сделать непосредственно: мы имеем в случае $t=q=1$

$$
\begin{array}{ll}
R=[1]=\square: & P=1-A^{2}\{A\}^{2}, \\
R=[2]: & P=\left(1-A^{2}\{A\}^{2}\right)^{2}=1-2 A^{2}\{A\}^{2}+A^{4}\{A\}^{4}, \\
R=[3]: & P=\left(\left(1-A^{2}\{A\}^{2}\right)^{3}=1-3 A^{2}\{A\}^{2}+3 A^{4}\{A\}^{4}-A^{6}\{A\}^{6}\right.
\end{array}
$$

в случае $t=q$

$$
\begin{array}{ll}
R=[1]=\square: & P=1-A^{2}\{A q\}\left\{\frac{A}{q}\right\}, \\
R=[2]: & P=1-[2]_{q} A^{2}\left\{A q^{2}\right\}\left\{\frac{A}{q}\right\}+q^{2} A^{4}\left\{A q^{3}\right\}\left\{A q^{2}\right\}\{A\}\left\{\frac{A}{q}\right\},
\end{array}
$$




$$
\begin{aligned}
R=[3]: \quad P= & 1-[3]_{q} A^{2}\left\{A q^{3}\right\}\left\{\frac{A}{q}\right\}+[3]_{q} q^{2} A^{4}\left\{A q^{4}\right\}\left\{A q^{3}\right\}\{A\}\left\{\frac{A}{q}\right\}- \\
& -q^{6} A^{6}\left\{A q^{5}\right\}\left\{A q^{4}\right\}\left\{A q^{3}\right\}\{A q\}\{A\}\left\{\frac{A}{q}\right\} ;
\end{aligned}
$$

в случае $t \neq q$

$$
\begin{aligned}
R=[1]=\square: \quad P & =1-\frac{A^{2} q}{t}\{A q\}\left\{\frac{A}{t}\right\}, \\
R=[2]: \quad P & =1-[2]_{q}\left(\frac{A^{2} q}{t}\right)\left\{A q^{2}\right\}\left\{\frac{A}{t}\right\}+q^{2}\left(\frac{A^{2} q}{t}\right)^{2}\left\{A q^{3}\right\}\left\{A q^{2}\right\}\left\{\frac{A q}{t}\right\}\left\{\frac{A}{t}\right\}, \\
R=[3]: \quad P= & 1-[3]_{q}\left(\frac{A^{2} q}{t}\right)\left\{A q^{3}\right\}\left\{\frac{A}{t}\right\}+ \\
& +[3]_{q} q^{2}\left(\frac{A^{2} q}{t}\right)^{2}\left\{A q^{4}\right\}\left\{A q^{3}\right\}\left\{\frac{A q}{t}\right\}\left\{\frac{A}{t}\right\}- \\
& -q^{6}\left(\frac{A^{2} q}{t}\right)^{3}\left\{A q^{5}\right\}\left\{A q^{4}\right\}\left\{A q^{3}\right\}\left\{\frac{A q^{2}}{t}\right\}\left\{\frac{A q}{t}\right\}\left\{\frac{A}{t}\right\} .
\end{aligned}
$$

Эти формулы иллюстрируют изменение как дифференциалов, так и $G$-коэффициентов. Альтернативное представление с расширенным набором дифференциалов вместо квантовых биномиальных коэффициентов можно найти в работах [17], [20].

Для общего (анти)симметрического представления имеются буквально такие же формулы, как и для узла-“восьмерки" $4_{1}$ :

$$
\begin{gathered}
P_{[r]}^{3_{1}}=1+\sum_{j=1}^{r} G_{j}^{3_{1}} \frac{[r]_{q} !}{[j]_{q} ![r-j]_{q} !} \prod_{i=0}^{j-1} D_{0}^{r+j} D_{1}^{j}, \\
P_{\left[1^{r}\right]}^{3_{1}}=1+\sum_{j=1}^{r} \bar{G}_{j}^{3_{1}} \frac{[r]_{t} !}{[j]_{t} ![r-j]_{t} !} \prod_{i=0}^{j-1} D_{r+j}^{0} D_{j}^{1} .
\end{gathered}
$$

Только на этот раз вместо выражения (3.6) мы имеем (3.8):

$$
\begin{aligned}
& G_{j}^{3_{1}}=(-1)^{j} A^{2 j} q^{j(2 j-1)} t^{j(2 j-3)}=\left(-\frac{A^{2} q}{t}\right)^{j} q^{2 j(j-1)}, \\
& \bar{G}_{j}^{3_{1}}=(-1)^{j} A^{2 j} q^{-j(2 j-3)} t^{-j(2 j-1)}=\left(-\frac{A^{2} q}{t}\right)^{j} t^{-2 j(j-1)} .
\end{aligned}
$$

Наконец, соотношения (3.5) между производящими функциями остаются теми же,

$$
\pi_{[r]}^{3_{1}}(z)=\left(\pi_{\square}^{3_{1}}(z)\right)_{q}^{r}, \quad \bar{\pi}_{\left[1^{r}\right]}^{3_{1}}(z)=\left(\bar{\pi}_{\square}^{3_{1}}(z)\right)_{t}^{r},
$$

но сами производящие функции должны быть слегка модифицированы - они содержат новую величину, оператор дилатации $\hat{\delta}_{q}: A \rightarrow q A$, так что

$$
\begin{gathered}
\pi_{[r]}^{3_{1}}(z)=1+\sum_{j=0}^{r} G_{j}^{3_{1}}\left(A^{2} \hat{\delta}_{q}\right) \frac{[r]_{q} !}{[j]_{q} ![r-j]_{q} !} z^{2 j}, \\
\bar{\pi}_{\left[1^{r}\right]}^{3_{1}}(z)=1+\sum_{j=0}^{r} \bar{G}_{j}^{3_{1}}\left(A^{2} \hat{\delta}_{-1 / t}\right) \frac{[r]_{q} !}{[j]_{q} ![r-j]_{q} !} z^{2 j} .
\end{gathered}
$$

В такой форме эти формулы остаются верными для всех твистованных узлов. 
В случае узла-“восьмерки" 41 со всеми координатами $G=1$ суперполиномы, по крайней мере во всех (анти)симметрических представлениях $R$, описывались не зависящим от $R$ перемешиванием набора дифференциалов $D[I]$, т. е. тем, что мы назвали операция $I_{R}$. Но уже для трилистника этого недостаточно: нужно добавить к известной операции $I_{R}$ операцию ${ }_{R}$, действующую на коэффициенты. По крайней мере для (анти)симметрических представлений, она сводится к последовательности операций $\circ_{R \rightarrow R^{\prime}}$ приклеивания клеток, более того, в этом случае достаточно рассмотреть $\circ_{r}=\circ_{[r] \rightarrow[r+1]}$ и $\bar{\circ}_{r}=\circ_{\left[1^{r}\right] \rightarrow\left[1^{r+1}\right]}$, действующие на мономы от $A^{2}$. Из приведенных формул ясно, что

$$
A^{2} \circ_{r} q^{r(r-1)} A^{2 r}=q^{r(r+1)} A^{2 r+2},
$$

следовательно,

$$
A^{2} \circ_{r} A^{2 r}=q^{2 r} A^{2 r+2}, \quad A^{2} \bar{\circ}_{r} A^{2 r}=t^{-2 r} A^{2 r+2} .
$$

Разностное уравнение (3.7) для трилистника остается почти тем же:

$$
P_{[r+1]}^{3_{1}}(A)-P_{[r]}^{3_{1}}(A)=\left\{A q^{2 r+1}\right\}\left\{\frac{A}{t}\right\} G_{\square}^{3_{1}}(A) P_{[r]}^{3_{1}}(q A) .
$$

Единственная разница состоит в наличии множителя $G_{\square}^{3_{1}}(A)=A^{2}$ в правой части равенства, в то время как для узла-"восьмерки" этот множитель $G_{\square}^{4_{1}}(A)=1$. Было бы естественно ожидать, что здесь появится операция свертки о, но равенство (3.13) подразумевает, что в данном частном случае она полностью заменяется тем же сдвигом $A \rightarrow q A$, который адекватно описывает операцию $I_{[r]}$ на наборе подходящих дифференциалов.

3.3. Твистованные узлы. В данном пункте мы опираемся на работы [17], [21]. Общая конструкция, которая применима ко всем твистованным узлам, непосредственно обобщает подход, описанный в п. 3.1 и 3.2. Основная идея - разложить полиномы в (анти)симметрических представлениях по тому же набору дифференциалов типа дифференциалов ДГР, как в соотношениях (3.1), (3.2) и (3.10). Для любого твистованного узла $\mathcal{K}$ во всех (анти)симметрических представлениях мы имеем

$$
\begin{gathered}
P_{[r]}^{\mathcal{K}}=1+\sum_{j=1}^{r} G_{j}^{\mathcal{K}} \frac{[r]_{q} !}{[j]_{q} ![r-j]_{q} !} \prod_{i=0}^{j-1} D_{0}^{r+j} D_{1}^{j}, \\
P_{\left[1^{r}\right]}^{\mathcal{K}}=1+\sum_{j=1}^{r} \bar{G}_{j}^{\mathcal{K}} \frac{[r]_{t} !}{[j]_{t} ![r-j]_{t} !} \prod_{i=0}^{j-1} D_{r+j}^{0} D_{j}^{1},
\end{gathered}
$$

где бесконечный набор коэффициентов $G_{j}^{\mathcal{K}}$ зависит от узла, но не от представления.

Поскольку твистованные узлы нумеруются одним параметром $k \in \mathbb{Z} \backslash 0$, в остальной части этого пункта мы обозначаем $G_{j}^{\mathcal{K}}$ как $G_{j}^{(k)}$. На уровне специальных полиномов все коэффициенты $G_{j}^{(k)}$ тривиальны и согласно правилу (3.11) являются простыми степенями коэффициента $G_{1}^{(k)}$ :

$$
\left.G_{j}^{(k)}\right|_{q=1}=\left(\left.G_{1}^{(k)}\right|_{q=1}\right)^{j} .
$$


Чтобы поднять это разложение на уровень полиномов ХОМФЛИ, необходимо обеспечить естественную $q$-деформацию умножения коэффициентов $G_{j}^{(k)}$ таким способом, чтобы было выполнено равенство

$$
G_{j}^{(k)}=\left(G_{1}^{(k)}\right)^{\circ j}
$$

В противоположность трилистнику (см. формулы (3.12), (3.13)) для общих твистованных узлов мы не требуем, чтобы операция о была бинарной. Вместо этого мы только утверждаем, что она универсальна для всех твистованных узлов и линейна по индексу $j$. Мы определяем и обсуждаем эту операцию в п. 3.3.1 и 3.3.2.

Последний переход от полиномов ХОМФЛИ к суперполиномам в терминах разложения (3.14) совсем простой - делается замена переменных (3.9) в коэффициентах разложения $G_{j}^{(k)}$ и восстанавливается переменная $t$ в дифференциалах $D_{0}^{r+j} D_{1}^{j}$ (для антисимметрических представлений - в дифференциалах $\left.D_{r+j}^{0} D_{j}^{1}\right)$. Вся нетривиальная часть этой процедуры заключается в том, что мы должны изначально знать подходящий набор дифференциалов для твистованных узлов в симметрических и антисимметрических представлениях. Мы обсуждаем обобщение этого метода вне симметрических/антисимметрических представлений и твистованных узлов ниже.

3.3.1. Операция о 1 для твистованных узлов, билинейный случай. В соотношении (3.15) мы предположили, что все коэффициенты разложения $G_{j}^{\mathcal{K}}$ данного твистованного узла $\mathcal{K}$ получаются из коэффициента $G_{1}^{\mathcal{K}}$ с помощью некоторой подходящей операции о. Чтобы проиллюстрировать это утверждение, мы начнем с простейшего примера $G_{2}^{(k)}$ при $k>0$, а общая конструкция будет представлена далее в п. 3.3.2.

В случае $G_{2}^{\mathcal{K}}$ операция о просто билинейна; чтобы не спутать с мультилинейной операцией, мы обозначим ее как $\circ_{1}$. Имеем $G_{2}^{\mathcal{K}}=G_{1}^{\mathcal{K}} \circ_{1} G_{1}^{\mathcal{K}}$. Первые два коэффициента таковы:

$$
G_{1}^{(k)}=A \frac{1-A^{2 k}}{\{A\}}, \quad G_{2}^{(k)}=q A^{2}\left(\frac{1}{\{A\}\{A q\}}-[2]_{q} \frac{A^{2 k}}{\{A\}\left\{A q^{2}\right\}}+\frac{q^{4 k} A^{4 k}}{\{A q\}\left\{A q^{2}\right\}}\right) .
$$

Для операции $\circ_{1}$ имеем

$$
A^{2 m} \circ_{1} A^{2 n}= \begin{cases}q^{4 m-2} A^{2 m+2 n}, & m \leqslant n, \\ q^{4 n} A^{2 m+2 n}, & m>n .\end{cases}
$$

В обоих случаях степень параметра $q$ зависит от меньшей из двух степеней переменной $A$. Удобно использовать следующую таблицу умножения:

\begin{tabular}{|c|c|c|c|c|c|c|c|}
\hline$\circ_{1}$ & $A^{2}$ & $A^{4}$ & $A^{6}$ & $A^{8}$ & $A^{10}$ & $A^{12}$ & $\ldots$ \\
\hline$A^{2}$ & $q^{2}$ & $q^{2}$ & $q^{2}$ & $q^{2}$ & $q^{2}$ & $q^{2}$ & $q^{2}$ \\
\hline$A^{4}$ & $q^{4}$ & $q^{6}$ & $q^{6}$ & $q^{6}$ & $q^{6}$ & $q^{6}$ & $q^{6}$ \\
\hline$A^{6}$ & $q^{4}$ & $q^{8}$ & $q^{10}$ & $q^{10}$ & $q^{10}$ & $q^{10}$ & $q^{10}$ \\
\hline$A^{8}$ & $q^{4}$ & $q^{8}$ & $q^{12}$ & $q^{14}$ & $q^{14}$ & $q^{14}$ & $q^{14}$ \\
\hline$A^{10}$ & $q^{4}$ & $q^{8}$ & $q^{12}$ & $q^{16}$ & $q^{18}$ & $q^{18}$ & $q^{18}$ \\
\hline$A^{12}$ & $q^{4}$ & $q^{8}$ & $q^{12}$ & $q^{16}$ & $q^{20}$ & $q^{22}$ & $q^{22}$ \\
\hline$\vdots$ & $q^{4}$ & $q^{8}$ & $q^{12}$ & $q^{16}$ & $q^{20}$ & $q^{24}$ & $\ddots$ \\
\hline
\end{tabular}


3.3.2. Операция о для твистованных узлов, мультилинейный случай. В данном пункте мы определяем мультилинейную операцию, введенную в (3.15). Эта операция действует на градуированном пространстве полиномов от $A$ и сохраняет соответствующую градуировку, а на локусе $q=1$ она превращается в простое умножение полиномов от $A$. Однако операция, определенная здесь, никоим образом не является коммутативной или ассоциативной.

Чтобы определить мультилинейную операцию на полиномах от $A$, достаточно описать ее действие на степени переменной $A$. Пусть у нас имеется произведение $n$ мономов от $A$, степени которых мы обозначаем через $k_{1}, k_{2}, \ldots, k_{n}$. Так как наша операция сохраняет градуировку, результат пропорционален $A^{\sum_{i=1}^{n} k_{i}}$,

$$
\circ\left(A^{k_{1}}, A^{k_{2}}, \ldots, A^{k_{n}}\right)=C_{\mathbf{k}} A^{\sum_{i=1}^{n} k_{i}} .
$$

Коэффициент пропорциональности $C_{\mathbf{k}}=c_{\mathbf{k}}^{\mathrm{a}} c_{\mathbf{k}}^{\mathrm{f}}$ состоит из двух частей: $c_{\mathbf{k}}^{\mathrm{a}}-$ средняя степень параметра $q$, которая зависит от набора степеней, но не от перестановок, и так называемая "тонкая структура" $c_{\mathbf{k}}^{\mathrm{f}}$, которая зависит только от повторных перестановок, но не от точных значений степеней в наборе $\mathbf{k}=\left\{k_{1}, k_{2}, \ldots, k_{n}\right\}$.

Известные формулы для $G_{j}^{(k)}$ в случае полиномов для твистованных узлов в (анти)симметрических представлениях единственным образом определяют коэффициент $c_{\mathbf{k}}^{\mathrm{a}}$ как функцию от $\mathbf{k}$. В то же время для второго коэффициента мы можем только утверждать, что суммирование $c_{\mathbf{k}}^{\mathrm{f}}$ по всем повторным перестановкам в набоpe $\mathbf{k}$ дает нам некоторый $q$-деформированный полиномиальный коэффициент. Как возможную реализацию такой структуры можно взять

$$
c_{\mathbf{k}}^{\mathrm{f}}=q^{2 \#(\text { inversions })-\max \#(\text { inversions })},
$$

где \#(inversions) определяет число инверсий в повторных перестановках набора $\mathbf{k}$.

Средняя степень параметра $q$ намного интереснее. Как было сказано, она зависит только от значений $k_{1}, k_{2}, \ldots, k_{n}$, но не от перестановок, что можно естественно изобразить диаграммой Юнга $\mathbf{D}$ с $\sum_{i=1}^{n} k_{i}$ клетками. Пусть $\left\{k_{i_{1}}, k_{i_{2}}, \ldots, k_{i_{n}}\right\}$ - частично упорядоченный набор:

$$
k_{i_{1}} \geqslant k_{i_{2}} \geqslant \cdots \geqslant k_{i_{n}}
$$

где $k_{i_{r}}$ - длина $r$-й строки в диаграмме Юнга D. Этот частично упорядоченный набор может быть параметризован числами $k_{j_{1}}^{m_{1}}, k_{j_{2}}^{m_{2}} \ldots, k_{j_{l}}^{m_{l}}$, образующими строго упорядоченный набор

$$
k_{j_{1}}>k_{j_{2}}>\cdots>k_{j_{l}}
$$

с множественностями $m_{1}, m_{2}, \ldots, m_{l}, n=\sum_{j=1}^{l} m_{j}$. Обозначим соответствующую диаграмму Юнга множественностей $m_{i}$ с $n$ клетками через $\mathbf{d}$.

Мы утверждаем, что

$$
\begin{array}{llll}
c_{\mathbf{k}}^{\mathrm{a}}=q^{2 \nu(\mathbf{D})-n(n-1) / 2-\nu\left(\mathbf{d}^{\mathrm{T}}\right)}, & \text { если } & k_{i}>0 & \text { для всех } i, \\
c_{\mathbf{k}}^{\mathrm{a}}=q^{2 \nu(\mathbf{D})-n(n-1) / 2+\nu\left(\mathbf{d}^{\mathrm{T}}\right)}, & \text { если } & k_{i}<0 & \text { для всех } i,
\end{array}
$$

где $\nu(\mathbf{D})=\sum_{i}(i-1) \mathbf{D}_{i}$. Заслуживает упоминания, что в соотношении (3.19) все величины $\mathbf{D}_{i}$ понимаются как отрицательные. Чтобы прояснить эти обозначения, ниже мы представляем пример 4. 
Операция о имеет весьма интересные свойства. Вследствие соотношений (3.18), (3.19) она не зависит от конкретного значения максимальной степени переменной $A$ (минимальной степени в случае отрицательных твистованных узлов), когда эта степень имеет единичную множественность в наборе $\left\{k_{1}, k_{2}, \ldots, k_{n}\right\}$. Это также можно заметить из таблицы (3.16). Зависимость от $\mathbf{k}$ разбивается на три независимые части - от числа перестановок, от набора степеней и от множественностей степеней.

Наконец, мы представляем несколько различных примеров, чтобы проиллюстрировать определение операции о.

Пример 1. Начнем с базового примера $\circ\left(A^{2}, A^{2}, A^{2}\right)$, здесь $k_{1}=k_{2}=k_{3}=2$ и соответствующая диаграмма имеет вид

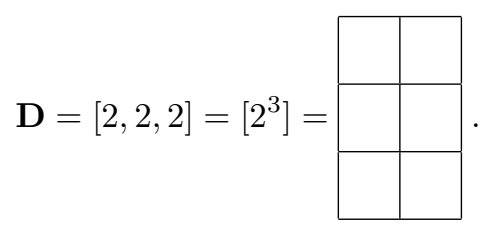

В этом случае имеется только одно значение степеней $k_{i}$ с множественностью 3. Таким образом, диаграмма множественностей $\mathbf{d}$ состоит из одной строки длины 3 :
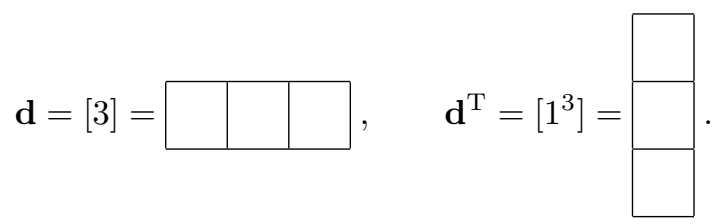

Теперь, используя формулу (3.18), мы получаем

$$
\circ\left(A^{2}, A^{2}, A^{2}\right)=c_{\{2,2,2\}}^{\mathrm{a}} A^{6}=q^{6} A^{6} .
$$

Данный пример не иллюстрирует равенство (3.17), поскольку в наборе $\{2,2,2\}$ нет нетривиальных параметров, и число инверсий всегда равно нулю. Это означает, что коэффициент $c_{\{2,2,2\}}^{\mathrm{f}}=1$ и не играет роли.

Пример 2. Мы продолжаем менее тривиальным примером, состоящим из различных степеней переменной $A$, а именно описываем все перестановки о $\left(A^{2}, A^{2}, A^{6}\right)$ :

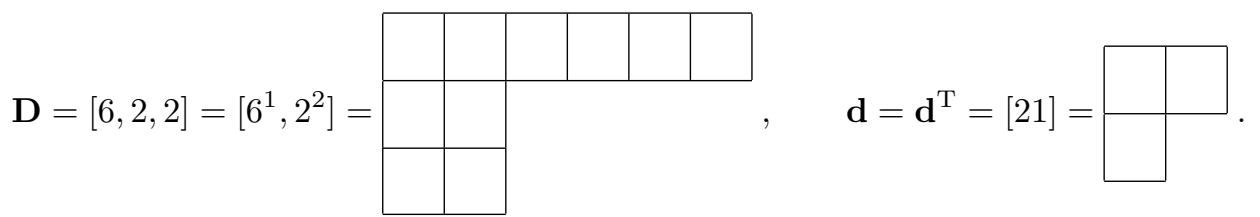

Снова используя формулу (3.18), мы получаем $c_{\{2,2,6\}}^{\mathrm{a}}=c_{\{2,6,2\}}^{\mathrm{a}}=c_{\{6,2,2\}}^{\mathrm{a}}=q^{8}$. Далее мы должны учесть равенство (3.17):

$$
c_{\{2,2,6\}}^{\mathrm{f}}=q^{-2}, \quad c_{\{2,6,2\}}^{\mathrm{f}}=1, \quad c_{\{6,2,2\}}^{\mathrm{f}}=q^{2} .
$$


Это в конце концов приводит нас к соотношениям

$$
\circ\left(A^{2}, A^{2}, A^{6}\right)=q^{6} A^{10}, \quad \circ\left(A^{2}, A^{6}, A^{2}\right)=q^{8} A^{10}, \quad \circ\left(A^{6}, A^{2}, A^{2}\right)=q^{10} A^{10} .
$$

ПримеР 3. Следующий пример включает нетривиальный полиномиальный коэффициент, когда берется сумма по всем перестановкам. С этой целью мы описываем все перестановки о $\left(A^{2}, A^{2}, A^{4}, A^{6}\right)$, которые включают три типа степеней. Здесь

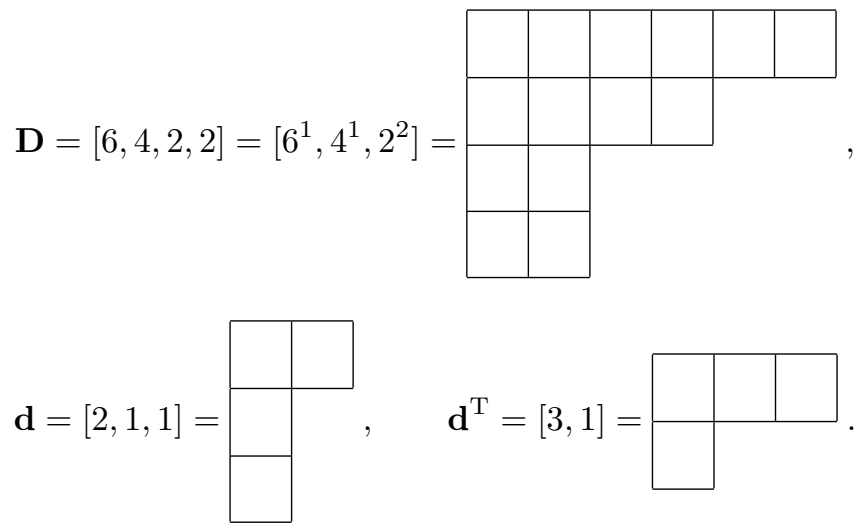

При этом $c_{\mathrm{k}}^{\mathrm{a}}=q^{21}$ и

$\begin{array}{lll}\circ\left(A^{2}, A^{2}, A^{4}, A^{6}\right)=q^{16} A^{14}, & \circ\left(A^{2}, A^{2}, A^{6}, A^{4}\right)=q^{18} A^{14}, & \circ\left(A^{2}, A^{4}, A^{2}, A^{6}\right)=q^{18} A^{14}, \\ \circ\left(A^{2}, A^{4}, A^{6}, A^{2}\right)=q^{20} A^{14}, & \circ\left(A^{2}, A^{6}, A^{2}, A^{4}\right)=q^{20} A^{14}, & \circ\left(A^{4}, A^{2}, A^{2}, A^{6}\right)=q^{20} A^{14}, \\ \circ\left(A^{2}, A^{6}, A^{4}, A^{2}\right)=q^{22} A^{14}, & \circ\left(A^{4}, A^{2}, A^{6}, A^{2}\right)=q^{22} A^{14}, & \circ\left(A^{4}, A^{6}, A^{2}, A^{2}\right)=q^{22} A^{14}, \\ \circ\left(A^{4}, A^{6}, A^{2}, A^{2}\right)=q^{24} A^{14}, & \circ\left(A^{6}, A^{2}, A^{4}, A^{2}\right)=q^{24} A^{14}, & \circ\left(A^{6}, A^{4}, A^{2}, A^{2}\right)=q^{26} A^{14} .\end{array}$

ПримеР 4. Последний пример иллюстрирует формулу (3.19) для отрицательных степеней переменной $A$ (это соответствует отрицательным твистованным узлам). Рассмотрим ० $\left(A^{-2}, A^{-2}, A^{-4}\right)$ и его перестановки. Здесь мы имеем

$$
\begin{aligned}
& \mathbf{D}=[-4,-2,-2]=\left[-4^{1},-2^{2}\right]=\begin{array}{|l|l|l|l|}
\hline- & - & - & - \\
\hline- & - \\
\hline
\end{array} \quad, \quad \nu(\mathbf{D})=-6, \\
& \mathbf{d}=\mathbf{d}^{\mathrm{T}}=[21]=\begin{array}{l|l|}
\hline &
\end{array}, \quad \nu\left(\mathbf{d}^{\mathrm{T}}\right)=1 .
\end{aligned}
$$


В результате $c_{\mathbf{k}}^{\mathrm{a}}=q^{-14}$. Далее, учитывая равенство (3.17), мы получаем

$$
\circ\left(A^{-4}, A^{-2}, A^{-2}\right)=q^{-16}, \quad \circ\left(A^{-2}, A^{-4}, A^{-2}\right)=q^{-14}, \quad \circ\left(A^{-2}, A^{-2}, A^{-4}\right)=q^{-12} \text {. }
$$

3.4. Двухнитевые торические узлы. Двухнитевые торические узлы представляют собой первый пример, когда $Z$-разложение по дифференциалам существенно отличается от разложения по характерам. В случае разложения по характерам для всех двухнитевых узлов имеется элементарная формула для полинома ХОМФЛИ в фундаментальном представлении, содержащая два члена:

$$
H_{\square}^{[2,2 k+1]}=\frac{A^{2 k+1}}{\left\{q^{2}\right\}}\left(q^{-2 k-1}\{A q\}-q^{2 k+1}\left\{\frac{A}{q}\right\}\right) .
$$

C другой стороны, $Z$-разложение намного сложнее, оно напоминает по сложности альтернативное представление в терминах $2 k+1$ нитей (которое также имеет порядок $2 k$ по дифференциалам).

А именно, мы выводим формулу вида

$$
H_{\square}^{[2,2 k+1]}=1+\sum_{j=1} g_{\square \mid j}^{[2,2 k+1]} \prod_{i=0}^{j-1} D_{0}^{r+i} D_{1}^{i}
$$

с коэффициентами $g_{\square} \mid j$, которые будут выписаны ниже. Наглядно в духе схем из работы [8] это означает, что мы представляем “змею” с двумя концами суммой ромбов с одной добавленной точкой (см. рис. 1). Замечательным образом при подходящей (топологически инвариантной) нормировке эта добавленная точка является в точности единицей. На самом деле это, по-видимому, будет верно для всех узлов, не только торических: разложение по дифференциалам всегда начинается с единицы. Ромб в данном случае представляет собой в точности первый $Z$-фактор $D_{0}^{1} D_{1}^{0}$. Затем мы рассматриваем $g_{\square \mid 0}=1$ как остаток от деления на $D_{0}^{1} D_{1}^{0}$, вычитаем его из полинома ХОМФЛИ и делим остающуюся часть на $D_{0}^{1} D_{1}^{0}$. Следующие шаги менее наглядны, однако на каждом шаге мы берем соответствующий остаток и продолжаем с частным. Для полинома ХОМФЛИ торического узла $T^{2,5}$ в фундаментальном представлении, таким образом, мы имеем

$$
H_{\square}^{[2,5]}=1-D_{0}^{1} D_{1}^{0}\left(\frac{A^{2}}{q}[2]_{q}+A^{6} q^{2}-D_{0}^{2} D_{1}^{1} A^{4}\right)
$$

или, эквивалентно,

$$
g_{\square \mid 0}^{[2,5]}=1, \quad g_{\square \mid 1}^{[2,5]}=-\frac{A^{2}}{q}[2]_{q}-A^{6} q^{2}, \quad g_{\square \mid 1}^{[2,5]}=A^{4} .
$$

Далее в этом пункте мы предполагаем, что всюду $g_{\ldots \mid 0}=1$.

Причиной для представления простого полинома в весьма хитрой форме является связь этого разложения со старшими (анти)симметрическими представлениями и тот факт, что оно прямо ведет к реконструкции суперполиномов. 


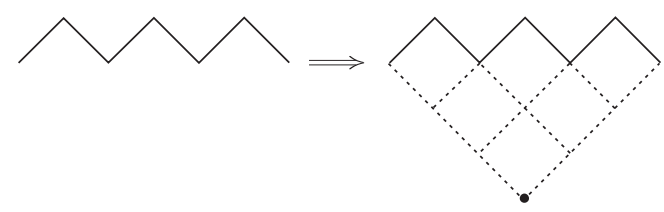

Рис. 1. Первый шаг разложения полинома ХОМФЛИ в случае торического узла $T_{\square}^{[2,5]}$.

Разложение (3.20) существует для всех двухнитевых узлов в симметрических представлениях и может быть сразу обобщено на антисимметрические представления:

$$
\begin{aligned}
& P_{[r]}^{[2,2 k+1]}=1+\sum_{j=1}^{k r} g_{[r], j}^{[2,2 k+1]} \prod_{i=0}^{j-1} D_{0}^{r+j} D_{1}^{j}, \\
& P_{\left[1^{r}\right]}^{[2,2 k+1]}=1+\sum_{j=1}^{k r} \bar{g}_{\left[1^{r}\right], j}^{[2,2 k+1]} \prod_{i=0}^{j-1} D_{r+j}^{0} D_{j}^{1} .
\end{aligned}
$$

Несколько первых примеров двухнитевых торических узлов даны в табл. 1-4. Заметим, что во всех представленных примерах зависимость $g_{i}$ от $i$ опять включена в $q$-биномиальный коэффициент. В случае фундаментального представления общая формула имеет следующий вид:

$$
g_{[1], j}^{[2,2 k+1]}=(-1)^{j} \sum_{i=0}^{k-1} q^{j^{2}-j k+2 i j+i} \frac{[k-i]_{q} !}{[j]_{q} ![k-i-j]_{q} !} \frac{[i+j-1]_{q} !}{[i]_{q} ![j-1]_{q} !}\left(\frac{A^{2} q}{t}\right)^{j+2 i} .
$$

Однако зависимость от представления не выделяется столь же замечательно, как в случае твистованных узлов. Как и в том случае, она полностью включена в комбинаторные коэффициенты, и это приводит нас к тому, что разложение (3.21) не представляет самую "тонкую структуру" инварианта узла. Соответствующие коэффициенты $g_{[r], j}^{2,2 k+1}$ все еще не являются искомыми координатами в пространстве полиномов узлов, но вместо этого они представляют собой сумму более "рафинированных" координат. Обсуждение этого явления и дальнейшие его обобщения содержатся в п. 3.6.

В нижеприведенных таблицах мы перечисляем несколько первых коэффициентов $g_{i}$ для нескольких первых двухнитевых узлов. Для краткости мы используем обозначение $a^{2}=-A^{2} q / t$. В терминах этой переменной (впервые использованной в статье [8]) все коэффициенты становятся положительными.

ТАБлицА 1. Торический узел $T^{2,3}$.

\begin{tabular}{|c|c|c|c|}
\hline$T^{2,3}$ & $\square$ & $\square \square$ & $\square \square \square$ \\
\hline$g_{1}$ & $a^{2}$ & $a^{2}[2]_{q}$ & $a^{2}[3]_{q}$ \\
\hline$g_{2}$ & & $a^{4} q^{2}$ & $a^{4} q^{2}[3]_{q}$ \\
\hline$g_{3}$ & & & $a^{6} q^{6}$ \\
\hline
\end{tabular}


ТАБЛицА 2. Торический узел $T^{2,5}$.

\begin{tabular}{|c|c|c|c|c|}
\hline$T^{2,5}$ & $\square$ & $\square \square$ & $\square \square \square$ & $\square \square \square \square$ \\
\hline$g_{1}$ & $\frac{a^{2}}{q}[2]_{q}+a^{6} q^{2}$ & $\frac{a^{2}}{q^{2}}[4]_{q}+a^{6} q^{4}[2]_{q}$ & $\frac{a^{2}}{q^{3}}[6]_{q}+a^{6} q^{6}[3]_{q}$ & $\frac{a^{2}}{q^{4}}[8]_{q}+a^{6} q^{8}[4]_{q}$ \\
\hline$g_{2}$ & $a^{4}$ & $\begin{array}{c}\frac{a^{4}}{q^{2}} \frac{[3]_{q}[4]_{q}}{[2]_{q}}+a^{8} q^{6}[2]_{q}[2]_{q}+ \\
+a^{12} q^{14}\end{array}$ & $\begin{array}{c}\frac{a^{4}}{q^{4}} \frac{[5]_{q}[6]_{q}}{[2]_{q}}+a^{8} q^{7}[4]_{q}[3]_{q}+ \\
+a^{12} q^{18}[3]_{q}\end{array}$ & $\begin{array}{c}\frac{a^{4}}{q^{6}} \frac{[7]_{q}[8]_{q}}{[2]_{q}}+a^{8} q^{8}[4]_{q}[6]_{q}+ \\
+a^{12} q^{22} \frac{[3]_{q}[4]_{q}}{[2]_{q}}\end{array}$ \\
\hline$g_{3}$ & & $a^{6}[4]_{q}+a^{10} q^{10}[2]_{q}$ & $\begin{array}{l}\frac{a^{6}}{q^{3}} \frac{[4]_{q}[5]_{q}[6]_{q}}{[2]_{q}[3]_{q}}+a^{10} q^{10} \frac{[3]_{q}^{2}[4]_{q}}{[2]_{q}}+ \\
+a^{14} q^{23}[2]_{q}[3]_{q}+a^{18} q^{36}\end{array}$ & $\begin{array}{c}\frac{a^{6}}{q^{6}} \frac{[6]_{q}[7]_{q}[8]_{q}}{[2]_{q}[3]_{q}}+a^{10} q^{10} \frac{[4]_{q}[5]_{q}[6]_{q}}{[2]_{q}}+ \\
+a^{14} q^{26} \frac{[3]_{q}[4]_{q}^{2}}{[2]_{q}}+a^{18} q^{42}[4]_{q}\end{array}$ \\
\hline$g_{4}$ & & $a^{8} q^{4}$ & $\begin{aligned} a^{8} \frac{[5]_{q}[6]_{q}}{[2]_{q}} & +a^{12} q^{15}[3]_{q}[4]_{q}+ \\
& +a^{16} q^{30}[3]_{q}\end{aligned}$ & $\begin{array}{l}\frac{a^{8}}{q^{4}} \frac{[5]_{q}[6]_{q}[7]_{q}[8]_{q}}{[2]_{q}[3]_{q}[4]_{q}}+a^{12} q^{14} \frac{[4]_{q}^{2}[5]_{q}[6]_{q}}{[2]_{q}[3]_{q}}+ \\
+a^{16} q^{32} \frac{[3]_{q}^{2}[4]_{q}^{2}}{[2]_{q}^{2}}+a^{20} q^{50}[2]_{q}[4]_{q}+a^{24} q^{68}\end{array}$ \\
\hline$g_{5}$ & & & $a^{10} q^{5}[6]_{q}+a^{14} q^{22}[3]_{q}$ & $\begin{array}{c}a^{10} \frac{[6]_{q}[7]_{q}[8]_{q}}{[2]_{q}[3]_{q}}+a^{14} t^{14} q^{20} \frac{[4]_{q}[5]_{q}[6]_{q}}{[2]_{q}}+ \\
+a^{18} q^{40} \frac{[3]_{q}[4]_{q}^{2}}{[2]_{q}}+a^{22} q^{60}[4]_{q}\end{array}$ \\
\hline$g_{6}$ & & & $a^{12} q^{12}$ & $\begin{array}{c}a^{12} q^{6} \frac{[7]_{q}[8]_{q}}{[2]_{q}}+a^{16} q^{28}[4]_{q}[6]_{q}+ \\
+a^{20} q^{50} \frac{[3]_{q}[4]_{q}}{[2]_{q}}\end{array}$ \\
\hline$g_{7}$ & & & & $a^{14} q^{14}[8]_{q}+a^{18} q^{38}[4]_{q}$ \\
\hline$g_{8}$ & & & & $a^{16} q^{24}$ \\
\hline
\end{tabular}


ТАБлицА 3. Торический узел $T^{2,7}$.

\begin{tabular}{|c|c|c|c|}
\hline$T^{2,7}$ & $\square$ & $\square$ & $\square \square \square$ \\
\hline$g_{1}$ & $\frac{a^{2}}{q^{2}}[3]_{q}+a^{6} q[2]_{q}+a^{10} q^{4}$ & $\frac{a^{2}}{q^{4}}[6]_{q}+a^{6} q^{2}[4]_{q}+a^{10} q^{8}[2]_{q}$ & $\frac{a^{2}}{q^{6}}[9]_{q}+a^{6} q^{3}[6]_{q}+a^{10} q^{12}[3]_{q}$ \\
\hline$g_{2}$ & $\frac{a^{4}}{q^{2}}[3]_{q}+a^{8} q^{3}[2]_{q}$ & $\frac{a^{4}}{q^{6}} \frac{[5]_{q}[6]_{q}}{[2]_{q}}+a^{8} q^{2}[4]_{q}^{2}+$ & $\frac{a^{4}[8]_{q}[9]_{q}}{q^{10}}+a^{8} q[6]_{q}[7]_{q}+$ \\
& $+a^{12} q^{10}\left(1+[3]_{q}^{2}\right)+$ & $+a^{12} q^{12} \frac{[3]_{q}[4]_{q}[5]_{q}}{[2]_{q}}+$ \\
& & $+a^{16} q^{18}[2]_{q}^{2}+a^{20} q^{26}$ & $+a^{16} q^{23}[3]_{q}[4]_{q}+a^{20} q^{34}[3]_{q}$ \\
\hline$g_{3}$ & $a^{6}$ & $\frac{a^{6}[4]_{q}[5]_{q}[6]_{q}}{q^{6}}+a^{10} q^{4} \frac{[3]_{q}[4]_{q}^{2}}{[2]_{q}}+$ & \\
\hline$g_{4}[3]_{q}$ & $+a^{14} q^{14}\left([2]_{q}+[4]_{q}[3]_{q}\right)+a^{18} q^{24}[4]_{q}$ & \\
\hline$g_{5}$ & & $\frac{a^{8}}{q^{4}} \frac{[5]_{q}[6]_{q}}{[2]_{q}}+a^{12} q^{8}[4]_{q}^{2}+a^{16} q^{20} \frac{[3]_{q}[4]_{q}}{[2]_{q}}$ & \\
\hline$g_{6}$ & & $a^{10}[6]_{q}+a^{14} t^{14} q^{14}[4]_{q}$ & \\
\hline
\end{tabular}


ТАБлицА 4. Торический узел $T^{2,9}$.

\begin{tabular}{|c|c|c|}
\hline$T^{2,9}$ & $\square$ & $\square \square$ \\
\hline$g_{1}$ & $\frac{a^{2}}{q^{3}}[4]_{q}+a^{6}[3]_{q}+a^{10} q^{3}[2]_{q}+a^{14} q^{6}$ & $\frac{a^{2}}{q^{6}}[8]_{q}+a^{6}[6]_{q}+a^{10} q^{6}[4]_{q}+a^{14} q^{12}[2]_{q}$ \\
\hline$g_{2}$ & $\frac{a^{4}}{q^{4}} \frac{[3]_{q}[4]_{q}}{[2]_{q}}+a^{8} q[3]_{q}[2]_{q}+a^{12} q^{6}[3]_{q}$ & \\
\hline$g_{3}$ & $\frac{a^{6}}{q^{3}}[4]_{q}+a^{10} q^{4}[3]_{q}$ & \\
\hline$g_{4}$ & $a^{8}$ & \\
\hline
\end{tabular}

3.5. Трехнитевый торический узел [3,4]. В трехнитевом случае $Z$-разложение можно получить, используя замечательный новый трюк, который оказывается тесно связанным с новой градуировкой из работы [24].

Рассмотрим разность

$$
P^{[3, k]}-P^{[2,2 k-1]}=p^{(k)}, \quad k \neq 0(\bmod 3),
$$

и получим ее $Z$-разложение. Оказывается, что, в то время как для двухнитевых узлов в симметрическом представлении параметры $g^{[2,2 k+1]}$ в действительности являются функциями только двух переменных, а именно параметра $q$ и произведения $a^{2} \equiv-A^{2} q / t$, в разности $p^{(k)}$ соответствующий коэффициент имеет одну дополнительную степень величины $q / t$. Таким образом, естественное масштабирование (связанное с четвертой градуировкой из работы [24])

$$
A \rightarrow A \sigma, \quad t \rightarrow t \sigma^{2}
$$

не влияет на $g$-коэффициенты для $P^{[2,2 k+1]}$ и для твистованных узлов в (анти)симметрических представлениях, но влияет на эти коэффициенты для $p^{(k)}$.

В фундаментальном представлении мы имеем для разности $p^{(k)}$ весьма простую формулу

$$
p^{(k)}=D_{0}^{1} D_{1}^{0}\left(\frac{(-1)^{k+1} a^{2 k}}{(-t / q)^{k^{*}}} \sum_{j=1}^{k^{*}} \frac{[k-3 j]_{q t}}{(-t / q)^{1-j}}\right), \quad k \neq 0(\bmod 3), \quad k^{*}=\left\lfloor\frac{k-1}{3}\right\rfloor,
$$

которая в простейшем случае $k=4$ дает

$$
P_{\square}^{[3,4]}-P_{\square}^{[2,7]}=\frac{q}{t}\left(\frac{A^{2} q}{t}\right)^{4} D_{0}^{1} D_{1}^{0} .
$$

Теперь, переходя к старшим симметрическим представлениям, нужно разложить соответствующую разность $p^{(k)}$ по тому же базису мультидифференциалов, который мы использовали для твистованных и двухнитевых торических узлов. Это разложение оказывается очень тесно связанным со свойством факторизации специальных полиномов. На уровне специальных полиномов $(q=t=1)$ мы имеем для произвольного узла $\mathcal{K}$ и представления $R$

$$
\mathcal{H}_{R}^{\mathcal{K}}=\left(\mathcal{H}_{\square}^{\mathcal{K}}\right)^{|R|}, \quad \mathcal{H}_{R}^{\mathcal{K}} \equiv P_{R}^{\mathcal{K}},
$$


где $\pi_{k}=\left.p^{(k)}\right|_{q=t=1}$. В работах [14], [29] было предложено расширить соотношение (3.23) на случай общих значений $t \neq 1$ в случае симметрических представлений и на случай общих значений $q \neq 1$ в случае антисимметрических ${ }^{5)}$, т. е.

$$
\left.P_{[r]}^{\mathcal{K}}(A, q, t)\right|_{q=1}=\left(\left.P_{\square}^{\mathcal{K}}(A, q, t)\right|_{q=1}\right)^{r} .
$$

Далее в этом разделе мы ограничиваемся симметрическими представлениями, антисимметрический случай совершенно аналогичен. Предположим, что существует разложение по той же паре дифференциалов, что и в случаях двухнитевых торических и твистованных узлов в симметрических представлениях:

$$
P_{[r]}^{\mathcal{K}}=1+\sum_{j=1}^{r} G_{[r], j}^{\mathcal{K}} \prod_{i=0}^{j-1} D_{0}^{r+j} D_{1}^{j} .
$$

Здесь нужно заметить, что последовательность пар дифференциалов, использованная для того чтобы разложить полиномы в симметрических представлениях, превращается в простую геометрическую прогрессию при $q=1$; действительно,

$$
\left(\prod_{i=0}^{j-1} D_{0}^{r+i} D_{1}^{i}\right)_{q=1}=\left(\{A\}\left\{\frac{A}{t}\right\}\right)^{j}
$$

таким образом, $\left.P_{[r]}^{\mathcal{K}}(A, q, t)\right|_{q=1}$ может рассматриваться как значение на массовой оболочке производящей функции коэффициентов разложения $G_{[r], j}^{\mathcal{K}}$ при $q=1$ :

$$
f_{[r]}^{\mathcal{K}}(\delta)=f_{\square}^{\mathcal{K}}(\delta)=\left.\sum_{j} \delta^{j} G_{[r], j}^{\mathcal{K}}(A, q, t)\right|_{q=1},
$$

на массовой оболочке

$$
\delta_{0}=\{A\}\left\{\frac{A}{t}\right\},\left.\quad P_{[r]}^{\mathcal{K}}(A, q, t)\right|_{q=1}=f_{[r]}^{\mathcal{K}}\left(\delta_{0}\right) .
$$

Если теперь учесть формулу (3.22), то на локусе $q=1$ мы имеем

$$
f_{[r]}^{[3,4]}(\delta)=\left(f_{\square}^{[2,7]}(\delta)+\frac{A^{8}}{t^{9}} \delta\right)^{r}=\sum_{i=0}^{r} \frac{r !}{i !(r-i) !}\left(f_{\square}^{[2,7]}(\delta)\right)^{i}\left(\frac{A^{8}}{t^{9}} \delta\right)^{r-i} .
$$

Ответ для суперполиномов представляет собой $q$-деформацию формулы (3.24). Основная идея состоит в том, чтобы описать отдельно $q$-деформацию дифференциалов и $q$-деформацию коэффициентов $G_{[r], j}^{\mathcal{K}}$. Что касается дифференциалов, кажется естественным сделать следующую подстановку, зависящую от представления $[r]$ :

$$
\delta^{j} \rightarrow \prod_{i=0}^{j-1} D_{0}^{r+i} D_{1}^{i}
$$

Чтобы описать $q$-деформацию закона умножения для коэффициентов, нужно рассмотреть $P_{[2]}^{[3,4]}$ как возмущение $P_{[2]}^{[2,7]}$, тогда имеем для разности $G_{[2]}^{[3,4]}-G_{[2]}^{[2,7]}$ peзультаты, отраженные в табл. 5 .

5)Эта гипотеза была впервые выдвинута в работе [14] (для любых диаграмм Юнга) только для суперполиномов торических узлов. Случай более общих узлов в произвольных симметрических или антисимметрических представлениях впервые обсуждался в работе [29]. 
ТАБлицА $5 . \quad q$-Деформация $G_{[2], j}^{[3,4]}-G_{[2], j}^{[2,7]}$.

\begin{tabular}{|c|c|c|}
\hline$j$ & $\tilde{g}_{[2], j}^{3,4}$ & $\widetilde{G}_{[2], j}^{3,4}$ \\
\hline 1 & $-2 a^{8} t^{7}$ & $-a^{8} t^{7}[2]_{q}$ \\
\hline 2 & $6 a^{10} t^{9}+4 a^{14} t^{13}+a^{16} t^{14}+2 a^{18} t^{17}$ & $a^{10} t^{9} q[2]_{q}[3]_{q}+a^{14} t^{13} q^{10}\left([2]_{q}\right)^{2}+$ \\
& & $+a^{16} t^{14} q^{12}+a^{18} t^{17} q^{19}[2]_{q}$ \\
\hline 3 & $-6 a^{12} t^{11}-4 a^{16} t^{15}$ & $-a^{12} t^{11} q^{4}[2]_{q}[3]_{q}-a^{16} t^{15} q^{15}\left([2]_{q}\right)^{2}$ \\
\hline 4 & $2 a^{14} t^{13}$ & $a^{14} t^{13} q^{9}[2]_{q}$ \\
\hline
\end{tabular}

\section{6. Итог: $Z$-разложение в случае (анти)симметрических представле-} ний. В этом пункте мы подводим итоги нашего знакомства с $Z$-разложением полиномов узлов в случае симметрических и антисимметрических представлений. Наше основное утверждение касается базиса мультидифференциалов. А именно, мы имеем следующие результаты.

- Для всех узлов $\mathcal{K}$ полиномы узлов должны разлагаться по $D_{0}^{r+j} D_{1}^{j}$ для симметрических представлений и по $D_{r+j}^{0} D_{j}^{1}$ для антисимметрических,

$$
\begin{gathered}
P_{[r]}^{\mathcal{K}}=1+\sum_{j=1}^{s \mathcal{K}^{r}} g_{[r], j}^{\mathcal{K}} \prod_{i=0}^{j-1} D_{0}^{r+i} D_{1}^{i}, \\
P_{\left[1^{r}\right]}^{\mathcal{K}}=1+\sum_{j=1}^{s_{\mathcal{K}} r} \bar{g}_{\left[1^{r}\right], j}^{\mathcal{K}} \prod_{i=0}^{j-1} D_{r+i}^{0} D_{i}^{1},
\end{gathered}
$$

где $s_{\mathcal{K}}=1$ для твистованных узлов, $s_{\mathcal{K}}=k$ для двухнитевых торических узлов $T^{[2,2 k+1]}$ и т. д. Это утверждение проверено для твистованных узлов, для двухнитевых торических узлов и для торического узла $T^{3,4}$. Хотя оно не проверено непосредственно для других узлов, существует множество указаний на то, что это утверждение верно и в общем случае.

- В случае двухнитевых торических и твистованных узлов коэффициенты разложения $g_{[r], j}^{\mathcal{K}}$ и $g_{\left[1^{r}\right], j}^{\mathcal{K}}$ могут быть подняты с полиномов ХОМФЛИ до суперполиномов совершенно непосредственно. С этой целью нужно сделать следующие подстановки в коэффициенты разложения $g^{\mathcal{K}}$ :

$$
\begin{array}{lll}
A^{2} \rightarrow \frac{A^{2} q}{t}, & q \rightarrow q & \text { в случае симметрических представлений, } \\
A^{2} \rightarrow \frac{A^{2} q}{t}, & q \rightarrow t & \text { в случае антисимметрических представлений. }
\end{array}
$$

Тогда для того, чтобы генерировать полный суперполином, достаточно подставить эти коэффициенты $g^{\mathcal{K}}$ в $(3.25)$. Результат не получается из полиномов ХОМФЛИ простой заменой переменных, поскольку дифференциалы $t$-деформируются не в соответствии с (3.26). 
- Для твистованных узлов зависимость коэффициентов $g^{(k)}$ от представления может быть полностью отделена от зависимости от узла, а именно

$$
g_{[r], j}^{(k)}=G_{j}^{(k)} \frac{[r]_{q} !}{[j]_{q} ![r-j]_{q} !}, \quad \bar{g}_{\left[1^{r}\right], j}^{(k)}=\bar{G}_{j}^{(k)} \frac{[r]_{t} !}{[j]_{t} ![r-j]_{t} !} .
$$

В этих формулах все зависимости от представления включены в биномиальный коэффициент. Таким образом, координаты $G_{j}^{(k)}$ (координаты $\bar{G}_{j}^{(k)}$ ) параметризуют суперполиномы конкретных твистованных узлов во всех симметрических (антисимметрических) представлениях.

- В действительности все коэффициенты $G_{j}^{(k)}$ для твистованных узлов могут быть восстановлены из $G_{1}^{(k)}$ при помощи подходящей полилинейной операции о, описанной в п. 3.3.2:

$$
G_{j}^{(k)}=\left(G_{1}^{(k)}\right)^{\circ j}
$$

Эта операция обеспечивает естественную $q$-деформацию (в случае $\bar{G}$ это $t$-деформация) умножения полиномов от $A$. Редукция этой операции к простому умножению на локусе $q=t=1$ происходит благодаря свойству факторизации специальных полиномов.

Обсудим, наконец, причину, почему зависимость от симметрического представления $[r]$ (антисимметрического представления $\left[1^{r}\right]$ ) не выделяется из коэффициентов $g^{\mathcal{K}}$ для всех узлов $\mathcal{K}$ так же просто, как для твистованных узлов. Ключевым здесь является тот факт, что разложение (3.25) - не самая "тонкая" возможная структура полиномов узлов в симметрических (антисимметрических) представлениях: каждая пара дифференциалов $D_{0}^{r+j} D_{1}^{j}$ (соответственно $D_{r+j}^{0} D_{j}^{1}$ ), которую мы использовали в разложении (3.25), есть сумма пар дифференциалов "самой тонкой структуры" (см. формулы (3.3), (3.4)). Твистованные узлы принадлежат частному случаю, когда все коэффициенты разложения на уровне "самой тонкой структуры" связаны специальным образом, приводя к $D_{0}^{r+j} D_{1}^{j}$. Это частное свойство гарантирует разделение переменных в сумме и, таким образом, поднимает его до $g_{j}^{(k)}$.

Мы предполагаем, что по указанному базису пар дифференциалов "самой тонкой структуры" все полиномы узлов разлагаются наиболее естественным образом, т. е. коэффициенты разложения не зависят от представления. Однако это явление не очевидно на уровне (анти)симметрических представлений. Частные свойства наборов дифференциалов, которые мы использовали, чтобы разложить полиномы, здесь не позволяют нам восстановить “самую тонкую структуру". Решение этой проблемы лежит вне (анти)симметрических представлений.

Следующий шаг по направлению к подходящему базису дифференциалов "самой тонкой структуры" обсуждается в следующем разделе. Элементы этого базиса в произвольном представлении $R$ естественно ассоциируются со всеми подмножествами клеток диаграммы Юнга $R$. Когда $R$ имеет одну строку или столбец, сумма по всем подмножествам диаграммы Юнга сводится просто к сумме по числу элементов в множестве. В этом причина того, что выражения (3.25) являются суммами только по одному индексу. 


\section{4. ПРЕДСТАВЛЕНИЯ ОБЩЕГО ВИДА}

4.1. Соображения общего характера. Намного меньше пока известно об иерархии дифференциалов для представлений общего вида, т. е. для диаграмм Юнга с более чем одной строкой или столбцом. Единственный на сегодняшний день опубликованный результат - это статья [23], и до сих пор даже не были независимо проверены выдвинутые в этой работе гипотезы. Тем не менее в работах [17] и [24] имеются некоторые дополнительные свидетельства в пользу подхода, который мы кратко формулируем ниже.

- Члены разложения по дифференциалам естественно градуированы степенями переменной $A$ в соответствующем разложении специального полинома:

$$
\left.P_{R}^{\mathcal{K}}(A \mid q, t)\right|_{q=t=1}=\left(\sigma_{\square}^{\mathcal{K}}(A)\right)^{|R|},
$$

где

$$
\sigma_{\square}^{\mathcal{K}}(A)=1+\sum_{k} s_{k}(A)\{A\}^{2 k}, \quad s_{k}(1) \neq 0 .
$$

Рассмотрим разложение

$$
P_{R}^{\mathcal{K}}(A \mid q, t)=\sum_{k=0} p_{R}^{(k)}(A \mid q, t)
$$

где $p^{(k)}(A \mid q, t)$ стремится к нулю, как $\hbar^{2 k}$, когда $q=e^{\text {const. } \hbar}, t=e^{\hbar}, A=e^{N \hbar}$.

- Для всех узлов и представлений $p_{R}^{(0)}=1$ [17]. Это правильная нормировка, ассоциированная с топологическим оснащением, т. е. нормировка, которая обеспечивает независимость полиномов узлов от конкретных реализаций в виде кос. Заметим, что эта нормировка отличается от групповой, когда в вычислениях, скажем, полиномов ХОМФЛИ используется универсальная $\mathcal{R}$-матрица (это отвечает вертикальному оснащению).

- В идеале, член $p^{(k)}(A \mid q, t)$ является линейной по $k$ комбинацией $Z$-факторов $Z_{a \mid d}^{b \mid c}=\left\{A q^{a} / t^{b}\right\}\left\{A q^{c} / t^{d}\right\}$ :

$$
p^{(k)}(A \mid q, t)=\sum_{|I|=k} g[I] Z^{\otimes k}[I]
$$

с коэффициентами $g[I]$, которые могут зависеть от $\mathcal{K}, R$ и переменных $A, q, t$, но которые не обязаны обращаться в ноль в пределе $\hbar=0$.

- Коэффициенты $g[I]$ также в некотором смысле не зависят от представления и регулярно зависят от узла $\mathcal{K}$ в любой “естественной” серии узлов, связанных эволюцией любого типа.

- Для узла-“восьмерки" $4_{1}$ все неисчезающие коэффициенты $g[I]$ являются $q$-биномами.

- На практике это действительно так, в лучшем случае, для прямоугольных диаграмм. В общем случае может понадобиться нечто подобное " $\epsilon^{2}$-членам" из работы [23] (при $\epsilon=q-1 / q$ ), которые не выглядят столь же "регулярно", как члены с $Z$, и могут даже содержать нечетное число дифференциалов. Предположительно, $\epsilon^{2}$-члены появляются только для $k \geqslant 2$ и не влияют на линейные по $Z$ члены. 
- Когда $A=t^{N}$ или $A=q^{-N}$, полином узла сводится к полиному $R_{\text {red }}$ в меньшем представлении без одной строки или одного столбца соответственно. Эта редукция не нарушает градуировку - члены $p_{R}^{(k)}$ с данным $k$ сводятся к $p_{R_{\text {red }}}^{(k)}$ с тем же $k$ :

$$
p_{R}^{(k)}=\left.p_{R_{\text {red }}}^{(k)}\right|_{A=t^{N} \text { или } A=q^{-N}} .
$$

- В качестве нетривиального обобщения свойства редукции мы имеем разностные соотношения для полиномов узлов как функций представлений (см. п. 4.4 ниже), причем эти соотношения также не нарушают градуировку. Иногда (например, в случае (анти)симметрических представлений [20]) они прямо приводятся к рекуррентным уравнениям, достаточным, чтобы полностью фиксировать полиномы (с подходящими начальными условиями), хотя в общем случае этот результат еще не получен. Также неясно, какое отношение эти простые и перспективные соотношения имеют к изысканным (но практически удобным) рекурсиям в духе работ [21], [26], на которые часто ссылаются как на квантовые $\mathcal{A}$-полиномы.

- Добавочные градуировки, подобные использованной в работе [24], могут модифицировать дифференциалы и $Z$-факторы множеством способов, но они, по-видимому, не влияют на коэффициенты $g[I]$, которые мы предлагаем рассматривать как правильные координаты на пространстве узлов. В этом смысле может случиться так, что новые градуировки не дают новых инвариантов узлов по сравнению с набором $\{g[I]\}$. Однако они могут быть полезны для того, чтобы находить разложения по дифференциалам как таковые, что, как мы уже видели, является не вполне тривиальной задачей даже для (анти)симметрических представлений.

В остальной части этого раздела мы до некоторой степени иллюстрируем некоторые пункты из этого списка. Вопросу четвертой градуировки [24] посвящен отдельный раздел 5 .

\section{2. Линейные по $Z$ члены: самосогласованный анзац для узла $4_{1}$ в про-} извольном представлении. В настоящее время самосогласованная гипотеза уже известна в случае линейных по $Z$ членов произвольных раскрашенных полиномов ХОМФЛИ для узла-“восьмерки”. Для диаграмм $R$ с одним крюком она была сформулирована в статье [23], здесь мы расширяем ее на произвольные $R$. Данная гипотеза служит стартовой точкой для всех дальнейших расширений - на члены старших порядков по $Z$ и на другие узлы. Самосогласованность означает, что линейные по $Z$ члены замечательно редуцируются сами по себе, когда $A=t^{N}$ и $A=q^{-N}$. В соответствии с нашим списком гипотез в п. 4.1 линейные по $Z$ члены свободны от $\epsilon^{2}$-членов (здесь $\left.\epsilon=q-1 / q\right)$ и полностью определяются $Z$-факторами, которые в случае полиномов ХОМФЛИ удобно параметризуются следующим образом:

$$
Z_{i \mid j}^{(s)}=Z_{i+s \mid j-s}=\left\{A q^{i+s}\right\}\left\{A q^{s-j}\right\} .
$$

Эти странные обозначения подразумевают, что $Z_{i \mid j}$ "сдвинуты" на $q^{s}$, и сдвиг $s$ играет важную роль в формулировке нашего правила.

Правило состоит из двух частей. Во-первых, с каждой клеткой диаграммы Юнга $R$ мы ассоциируем $Z$-фактор почти такой же, как в формулах для диаграмм с одним крюком. Во-вторых, мы сдвигаем $Z$-фактор, и сдвиг в конкретной клетке 


\begin{tabular}{|c|c|c|c|c|c|c|c|c|c|}
\hline $\begin{array}{c}(0) \\
19,13\end{array}$ & $\begin{array}{c}(0) \\
17,13\end{array}$ & $\begin{array}{c}(0) \\
15,13\end{array}$ & $\begin{array}{c}(0) \\
13,13\end{array}$ & $\begin{array}{c}(2) \\
11,9\end{array}$ & $\begin{array}{l}(2) \\
9,9\end{array}$ & $\begin{array}{l}(2) \\
7,9\end{array}$ & $\begin{array}{l}(3) \\
5,7\end{array}$ & $\begin{array}{l}(4) \\
3,5\end{array}$ & $\begin{array}{l}(4) \\
1,5\end{array}$ \\
\hline $\begin{array}{c}(0) \\
19,11\end{array}$ & $\begin{array}{c}(0) \\
17,11\end{array}$ & $\begin{array}{c}(0) \\
15,11\end{array}$ & $\begin{array}{c}(0) \\
13,11\end{array}$ & $\begin{array}{c}(2) \\
11,7\end{array}$ & $\begin{array}{l}(2) \\
9,7\end{array}$ & $\begin{array}{l}(2) \\
7,7\end{array}$ & $\begin{array}{l}(3) \\
5,5\end{array}$ & $\begin{array}{l}(4) \\
3,3\end{array}$ & $\begin{array}{l}(4) \\
1,3\end{array}$ \\
\hline $\begin{array}{c}(0) \\
19,9\end{array}$ & $\begin{array}{c}(0) \\
17,9 \\
\end{array}$ & $\begin{array}{c}(0) \\
15,9\end{array}$ & $\begin{array}{c}(0) \\
13,9\end{array}$ & $\begin{array}{c}(2) \\
11,5\end{array}$ & $\begin{array}{l}(2) \\
9,5\end{array}$ & $\begin{array}{l}(2) \\
7,5\end{array}$ & $\begin{array}{l}(3) \\
5,3\end{array}$ & $\begin{array}{l}(4) \\
3,1\end{array}$ & $\begin{array}{l}(4) \\
1,1\end{array}$ \\
\hline $\begin{array}{l}(-2) \\
15,7\end{array}$ & $\begin{array}{l}(-2) \\
13,7\end{array}$ & $\begin{array}{l}(-2) \\
11,7\end{array}$ & $\begin{array}{l}(0) \\
9,7\end{array}$ & $\begin{array}{l}(2) \\
7,3\end{array}$ & $\begin{array}{l}(2) \\
5,3 \\
\end{array}$ & $\begin{array}{l}(2) \\
3,3 \\
\end{array}$ & $\begin{array}{l}(3) \\
1,1 \\
\end{array}$ & & \\
\hline $\begin{array}{l}(-3) \\
13,5 \\
\end{array}$ & $\begin{array}{l}(-3) \\
11,5\end{array}$ & $\begin{array}{c}(-3) \\
9,5 \\
\end{array}$ & $\begin{array}{c}(-1) \\
7,5\end{array}$ & $\begin{array}{l}(0) \\
5,1 \\
\end{array}$ & $\begin{array}{l}(0) \\
3,1 \\
\end{array}$ & $\begin{array}{l}(0) \\
1,1 \\
\end{array}$ & & & \\
\hline $\begin{array}{c}(-6) \\
7,3\end{array}$ & $\begin{array}{c}(-6) \\
5,3\end{array}$ & $\begin{array}{c}(-6) \\
3,3\end{array}$ & $\begin{array}{c}(-4) \\
1,3\end{array}$ & & & & & & \\
\hline $\begin{array}{c}(-6) \\
7,1\end{array}$ & $\begin{array}{c}(-6) \\
5,1\end{array}$ & $\begin{array}{c}(-6) \\
3,1\end{array}$ & $\begin{array}{c}(-4) \\
1,1\end{array}$ & & & & & & \\
\hline
\end{tabular}

Рис. 2. Линейные члены разложения по $Z$ для $R=[10,10,10,8,7,4,4]$ : каждая клетка диаграммы дает $Z$-фактор, показанный на рисунке.

зависит несколько более сложным образом от ее расположения в диаграмме $R$. Обе процедуры иллюстрируются для $R=[10,10,10,8,7,4,4]$ на рис. 2.

Пусть $\begin{gathered}(s) \\ i, j\end{gathered}$ - элемент рис. 2, он отвечает линейному члену $\propto Z_{i \mid j}^{(s)}=Z_{i+s \mid j-s}$. Индекс $i$ считает количество клеток справа от конкретной клетки, в то время как индекс $j$ считает количество клеток снизу по следующему правилу: $i$ равно удвоенному количеству клеток справа плюс единица, $j$ равно удвоенному количеству клеток снизу плюс единица. Сдвиг $s$ конструируется более хитрым способом. Расщепим диаграмму Юнга $Y$ на вложенные крюки $\Gamma_{i}$. Первая строка вместе с первым столбцом формируют первый крюк и т. д., $Y=\bigcup_{i} \Gamma_{i}$. Зафиксируем конкретную $n$-ю клетку, скажем, с длиной столбца $r_{n}$ и длиной строки $s_{n}$, сформируем поддиаграмму $Y_{n} \equiv \bigcup_{i \geqslant n} \Gamma_{i}$ и дополним ее до полного прямоугольника $B_{n}$ размера $r_{n} \times s_{n}$. Тогда сдвиг $s$ для любого элемента $\Gamma_{n}$ - это разность числа клеток в $B_{n} / Y_{n}$ снизу и справа от этого элемента.

4.3. Пример прямоугольных диаграмм: представление [22]. Данный пример показывает очень ясно, какого типа критерии можно использовать, чтобы найти походящее разложение по $Z$. А именно, один и тот же полином ХОМФЛИ для трилистника (полученный, к примеру, по формуле Россо-Джонса) может быть разложен множеством способов, из которых мы представляем три вместе с их аналогами для узла-“восьмерки" (его полином ХОМФЛИ можно найти в работах [24] и [30]).

ВеРсия 1:

$$
\begin{aligned}
H_{[22]}^{3_{1}}= & 1-A^{2}\left(Z_{1 \mid 1}+Z_{1 \mid 3}+Z_{3 \mid 1}+Z_{3 \mid 3}\right)+ \\
& +A^{4} Z_{2 \mid 2} D_{0}^{0}\left(q^{4} D_{0}^{2}+q^{-4} D_{0}^{-2}+D_{0}^{4}+D_{0}^{-4}+A^{-1}\left\{q^{2}\right\}^{2}\right)+ \\
& +A^{4} Z_{2 \mid 2} Z_{3 \mid 3}\left(\left(q^{2}+q^{-2}\right)-\left(q+q^{-1}\right)^{2} A^{2} Z_{1 \mid 1}+A^{4} Z_{1 \mid 1} Z_{2 \mid 2}\right), \\
H_{[22]}^{4_{1}}= & 1+\left(Z_{1 \mid 1}+Z_{1 \mid 3}+Z_{3 \mid 1}+Z_{3 \mid 3}\right)+Z_{2 \mid 2} D_{0}^{0}\left(D_{0}^{2}+D_{0}^{-2}+D_{0}^{4}+D_{0}^{-4}\right)+ \\
& +Z_{2 \mid 2} Z_{3 \mid 3}\left(2+\left(q+q^{-1}\right)^{2} Z_{1 \mid 1}+Z_{1 \mid 1} Z_{2 \mid 2}\right) .
\end{aligned}
$$


В этом случае полином $H_{[22]}^{4_{1}}$ выглядит более или менее приемлемо, но полином $H_{[22]}^{3_{1}}$ содержит $\epsilon^{2}$-член $\left\{q^{2}\right\}^{2} A^{3} D_{0}^{0} Z_{2 \mid 2}$. Линейные по $Z$ члены находятся в соответствии с гипотезой из п. 4.1.

ВЕРСИЯ 2:

$$
\begin{aligned}
H_{[22]}^{3_{1}}=1 & -[2]_{q}\left(q^{5}+q^{-5}\right) A^{2} Z_{2 \mid 2}+[3]_{q}\left(q^{4}+q^{-4}\right) A^{4} Z_{1 \mid 1} Z_{2 \mid 2}- \\
& -[2]_{q}^{2} A^{6} Z_{1 \mid 1} Z_{2 \mid 2} Z_{3 \mid 3}+A^{8} Z_{1 \mid 1} Z_{2 \mid 2}^{2} Z_{3 \mid 3}= \\
=1 & -[2]_{q}[2]_{q^{5}} A^{2} Z_{2 \mid 2}+[3]_{q}[2]_{q^{4}} A^{4} Z_{1 \mid 1} Z_{2 \mid 2}- \\
& -[2]_{q}^{2} A^{6} Z_{1 \mid 1} Z_{2 \mid 2} Z_{3 \mid 3}+A^{8} Z_{1 \mid 1} Z_{2 \mid 2}^{2} Z_{3 \mid 3}, \\
H_{[22]}^{4_{1}}=1+ & {[2]_{q}^{2}\left(q^{4}-q^{2}-1-q^{-2}+q^{-4}\right) Z_{2 \mid 2}+[3]_{q}[2]_{q^{2}} Z_{1 \mid 1} Z_{2 \mid 2}+} \\
+ & {[2]_{q}^{2} Z_{1 \mid 1} Z_{2 \mid 2} Z_{3 \mid 3}+Z_{1 \mid 1} Z_{2 \mid 2}^{2} Z_{3 \mid 3} . }
\end{aligned}
$$

Эти два разложения выглядят вполне хорошо с точки зрения выбора $Z$-факторов, в частности отсутствуют $\epsilon^{2}$-члены. Однако коэффициенты выглядят много хуже, особенно в случае $H_{[22]}^{4_{1}}$. Как иллюстрация этого подчеркнутый линейный по $Z$ член во второй формуле из (4.1) отличается от предположения в п. 4.1.

ВЕРСИЯ 3:

$$
\begin{aligned}
H_{[22]}^{3_{1}}=1 & -[2]_{q}^{2} A^{2} Z_{2 \mid 2}+[3]_{q} A^{4} Z_{2 \mid 2}\left(q^{2} Z_{3 \mid 1}+q^{-2} Z_{1 \mid 3}\right)- \\
& -[2]_{q}^{2} A^{6} Z_{1 \mid 1} Z_{2 \mid 2} Z_{3 \mid 3}+A^{8} Z_{1 \mid 1} Z_{2 \mid 2}^{2} Z_{3 \mid 3} \\
H_{[22]}^{4_{1}}=1 & +[2]_{q}^{2} Z_{2 \mid 2}+[3]_{q} Z_{2 \mid 2}\left(Z_{3 \mid 1}+Z_{1 \mid 3}\right)+ \\
& +[2]_{q}^{2} Z_{1 \mid 1} Z_{2 \mid 2} Z_{3 \mid 3}+Z_{1 \mid 1} Z_{2 \mid 2}^{2} Z_{3 \mid 3}
\end{aligned}
$$

Мы полагаем, что эти формулы являются правильными разложениями по $Z$ : в них нет $\epsilon^{2}$-членов (как и должно быть для [22], являющейся прямоугольной диаграммой), линейные по $Z$ члены находятся в соответствии с предположениями из п. 4.1, и другие коэффициенты также имеют приемлемый вид. Более того, мы ожидаем, что в случае узла $4_{1}$ коэффициенты можно на самом деле сделать равными единице: пользуясь тождествами, подобными $Z_{1 \mid 1}+Z_{1 \mid 3}+Z_{3 \mid 1}+Z_{3 \mid 3}=[2]_{q}^{2} Z_{2 \mid 2}$, запишем второе выражение в (4.2) как сумму $Z$-факторов по всем подмножествам диаграммы Юнга [22]. Это можно сделать несколькими способами, одна из возможных реализаций такова:

$$
\begin{aligned}
H_{[22]}^{4_{1}}=1 & +\left(Z_{1 \mid 1}+Z_{1 \mid 3}+Z_{3 \mid 1}+Z_{3 \mid 3}\right)+ \\
& +\left(Z_{0 \mid 2} Z_{1 \mid 3}+Z_{3 \mid 1} Z_{1 \mid 3}+Z_{3 \mid 3} Z_{1 \mid 3}+Z_{2 \mid 0} Z_{3 \mid 1}+Z_{1 \mid 1} Z_{3 \mid 3}+Z_{3 \mid 1} Z_{3 \mid 3}\right)+ \\
& +\left(Z_{1 \mid 1} Z_{1 \mid 3} Z_{3 \mid 1}+Z_{1 \mid 1} Z_{3 \mid 3} Z_{3 \mid 1}+Z_{1 \mid 3} Z_{3 \mid 3} Z_{3 \mid 1}+Z_{1 \mid 1} Z_{1 \mid 3} Z_{3 \mid 3}\right)+Z_{1 \mid 1} Z_{2 \mid 2}^{2} Z_{3 \mid 3} .
\end{aligned}
$$

4.4. Указания на возникновение рекуррентных соотношений. Один из первых базовых результатов о $Z$-разложении, недавно полученный в статье [20], это набор простых рекуррентных соотношений, подобных

$$
\begin{aligned}
P_{[r+1]}^{4_{1}}(A)-P_{[r]}^{4_{1}}(A) & =\left\{A q^{2 r+1}\right\}\left\{\frac{A}{t}\right\} P_{[r]}^{4_{1}}(q A), \\
P_{\left[1^{r+1}\right]}^{4_{1}}(A)-P_{\left[1^{r}\right]}^{4_{1}}(A) & =\{A q\}\left\{\frac{A}{t^{2 r+1}}\right\} P_{\left[1^{r}\right]}^{4_{1}}\left(\frac{A}{t}\right) .
\end{aligned}
$$


Эти выражения много проще, чем обычные квантовые $\mathcal{A}$-полиномы, но зато они не допускают никакой простой редукции к $A=t^{N}$ и $A=q^{-N}$, включая редукцию к полиномам Джонса (для $N=2$ ).

Обобщение на более сложные представления требует более глубокого исследования. Тем не менее некоторые нетривиальные факты уже известны для прямоугольных диаграмм Юнга: мы имеем для произвольных $r_{1}, r_{2}$ и $k$

$$
P_{\left[r_{1}^{k}\right]}-P_{\left[r_{2}^{k}\right]} \sim\left\{A q^{r_{1}+r_{2}}\right\}\left\{\frac{A}{t^{k}}\right\}=\mathcal{Z}_{r_{1}+r_{2} \mid k} .
$$

Однако коэффициент перед этим $Z$-фактором еще не идентифицирован подходящим образом и выражается через суперполиномы в некоторых других представлениях и с некоторым образом сдвинутыми аргументами. С другой стороны, пропорциональность такого типа, видимо, имеет место не только для узла-“восьмерки" $4_{1}$, но также для трилистника $3_{1}$ и, вероятно, для других узлов.

\section{5. Комментарий об отрицательных коэффициентах в торических су-} перполиномах. Поскольку трилистник является торическим узлом, все полиномы ХОМФЛИ даются формулой Россо-Джонса. Суперполиномы можно получить разными способами. К примеру, можно использовать тот факт, что трилистник одновременно является твистованным узлом и, таким образом, принадлежит общей серии с узлом 41 (для которого нетривиальные полиномы ХОМФЛИ получены в работе [30]); эти полиномы могут быть $t$-деформированы все сразу в духе работы [17].

Другой возможностью является использование суперполиномов, предложенных в статье [14]. Однако существует хорошо известная проблема, связанная с суперполиномами в старших представлениях и полученными таким способом: они, вообще говоря, не являются положительными полиномами в переменных $(a, t, T)$. В то же время в работе [24] можно найти несколько явных примеров улучшенных (положительных) суперполиномов трилистника для диаграмм Юнга с несколькими строками и столбцами. Суперполиномы, вычисленные по методу статьи [14], имеют отрицательные коэффициенты, если они раскрашены не прямоугольными диаграммами Юнга $\left[r^{s}\right]$. В случае прямоугольных диаграмм выражения для суперполиномов из статьи [14] совпадают с ответами из работы [24]. Единственный представленный в работе [24] пример вне прямоугольных диаграмм - это трилистник в представлении [21]. Сравнивая этот суперполином с суперполиномом, сконструированным в работе [14], мы обнаруживаем, что между ними имеется интересная связь. А именно, если переписать их в переменных $(a, t, T)$, то нужно взять набор всех степеней переменной $t$, которые входят в мономы с отрицательными коэффициентами. Будем далее ссылаться на такой набор степеней как на $\pi_{t}$. Применим следующую простую операцию ко всем мономам со степенями переменной $t$ из $\pi_{t}$ : заменяем коэффициент -1 на $T$ и делим мономы с положительными коэффициентами на $T^{2}$. Чтобы проиллюстрировать это правило, мы представляем таблицы коэффициентов (см. табл. 6 и 7) для двух вышеупомянутых полиномов. В этих таблицах для того, чтобы сравнивать два полинома, мы опустили несущественные общие множители. 
ТАБлицА 6. Коэффициенты суперполинома для трилистника $33_{1}$ в представлении [21] из статьи [14].

\begin{tabular}{|c|c|c|c|c|c|c|c|c|c|c|c|}
\hline & $t^{-10}$ & $t^{-8}$ & $t^{-6}$ & $t^{-4}$ & $t^{-2}$ & $t^{0}$ & $t^{2}$ & $t^{4}$ & $t^{6}$ & $t^{8}$ & $t^{10}$ \\
\hline$a^{6}$ & 0 & 0 & 0 & 0 & 0 & $T^{15}$ & 0 & 0 & 0 & 0 & 0 \\
\hline$a^{4}$ & 0 & 0 & $T^{8}$ & $T^{10}$ & $T^{10}$ & $-T^{10}+T^{12}$ & $T^{12}$ & $T^{14}$ & $T^{14}$ & 0 & 0 \\
\hline$a^{2}$ & $T^{3}$ & 0 & $2 T^{5}$ & $-T^{5}+T^{7}$ & $3 T^{7}$ & $-T^{7}+T^{9}$ & $3 T^{9}$ & $-T^{9}+T^{11}$ & $2 T^{11}$ & 0 & $T^{13}$ \\
\hline$a^{0}$ & 1 & 0 & $2 T^{2}$ & $-T^{2}$ & $2 T^{4}$ & $-T^{4}+T^{6}$ & $2 T^{6}$ & $-T^{6}$ & $2 T^{8}$ & 0 & $T^{10}$ \\
\hline
\end{tabular}

ТАБлицА 7. Коэффициенты суперполинома для трилистника 31 в представлении [21] из статьи [24].

\begin{tabular}{|c|c|c|c|c|c|c|c|c|c|c|c|}
\hline & $t^{-10}$ & $t^{-8}$ & $t^{-6}$ & $t^{-4}$ & $t^{-2}$ & $t^{0}$ & $t^{2}$ & $t^{4}$ & $t^{6}$ & $t^{8}$ & $t^{10}$ \\
\hline$a^{6}$ & 0 & 0 & 0 & 0 & 0 & $T^{13}$ & 0 & 0 & 0 & 0 & 0 \\
\hline$a^{4}$ & 0 & 0 & $T^{8}$ & $T^{8}$ & $T^{10}$ & $T^{11}+T^{10}$ & $T^{12}$ & $T^{12}$ & $T^{14}$ & 0 & 0 \\
\hline$a^{2}$ & $T^{3}$ & 0 & $2 T^{5}$ & $T^{6}+T^{5}$ & $3 T^{7}$ & $T^{8}+T^{7}$ & $3 T^{9}$ & $T^{10}+T^{9}$ & $2 T^{11}$ & 0 & $T^{13}$ \\
\hline$a^{0}$ & 1 & 0 & $2 T^{2}$ & $T^{3}$ & $2 T^{4}$ & $T^{5}+T^{4}$ & $2 T^{6}$ & $T^{7}$ & $2 T^{8}$ & 0 & $T^{10}$ \\
\hline
\end{tabular}




\section{Z-РАЗЛОЖЕНИЕ И ДОПОЛНИТЕЛЬНАЯ ГРАДУИРОВКА}

5.1. Введение дополнительных градуировок. Как мы уже объясняли выше, реконструкция $Z$-разложения из первых нескольких полиномов узлов неоднозначна. Следовательно, определить правильное $Z$-разложение заданного полинома - это все еще совершенно нетривиальная задача. Проблему можно, однако, упростить, введя новые градуировки, которые различают разные дифференциалы. На самом деле, для того чтобы сконструировать $Z$-разложение любого раскрашенного суперполинома, необходимо ввести бесконечно много новых градуировок, но для низших представлений нужны только несколько.

В данном разделе мы рассматриваем простейший случай одной добавочной градуировки, которая была введена в работе [24], и демонстрируем, как она возникает в рамках иерархии дифференциалов. Существует еще один аргумент для введения дополнительных градуировок - как мы уже упоминали в начале п. 3.5, она кажется совершенно необходимой после наблюдения Чередника [14], что раскрашенные торические суперполиномы могут не оказаться положительными вне прямоугольных представлений $\left[r^{s}\right]$. Выход (если эта проблема вообще существует, см. комментарий в п. 4.5 о том, как она решается в статье [24]), как предполагается, заключается в том, что в результате этой процедуры получается на самом деле эйлерова характеристика $t$-деформированного комплекса. В то же время нам требуется полином Пуанкаре этого комплекса, который зависит от дополнительной переменной $T$. Все эти условия вместе дают нам полином от четырех переменных $(A, q, t, T)$.

Мы предполагаем, что введение дополнительной градуировки в [24] тесно связано с историей об иерархии дифференциалов, но мы не уверены, что структуры, которые неявно подразумеваются в указанной работе, в точности совпадают с нашими. Ниже переменная дополнительной градуировки обозначена как $\sigma$ (мы объясним ее связь с переменными из статьи [24] позже в этом разделе). Мы утверждаем следующее.

- $\mathrm{B}$ терминах переменных $(A, q, t, \sigma)$ четвертую градуировку можно полностью алгоритмически реконструировать из $Z$-разложения. Строительные блоки $Z$-разложения - это пара дифференциалов $D[I]$ типа дифференциалов ДГР. Каждая пара состоит из одного дифференциала, который масштабируется как $A \rightarrow A / \sigma$, и другого дифференциала, который масштабируется как $A \rightarrow A \sigma$. В то же время все коэффициенты разложения $G_{R}^{\mathcal{K}}$ остаются неизменными. Эта гипотеза верна во всех примерах четырежды градуированных гомологий, представленных в работе [24]. Мы даем явное разложение для каждого из этих примеров в п. 5.2-5.5.

- Специфические свойства $Z$-разложения суперполиномов двухнитевых торических и твистованных узлов в симметрических и антисимметрических представлениях делают введение четвертой градуировки даже более тривиальным. Ключевой чертой этого разложения является то, что коэффициенты разложения $G_{[r], j}^{\mathcal{K}}$ или $G_{\left[1^{s}\right], j}^{\mathcal{K}}$ в действительности представляют собой полиномы только от двух из трех переменных, а именно

$$
\begin{aligned}
G_{[r], j}^{\mathcal{K}} & =G_{[r], j}^{\mathcal{K}}\left(\frac{A^{2} q}{t}, q\right), \\
G_{\left[1^{s}\right], j}^{\mathcal{K}} & =G_{\left[1^{s}, j\right]}^{\mathcal{K}}\left(\frac{A^{2} q}{t}, t\right)
\end{aligned}
$$


для узлов $\mathcal{K}$, являющихся либо двухнитевыми торическими, либо твистованными. Далее, заметим, что введение переменной $\sigma$ в дифференциалы, предложенное выше, проводится просто путем тривиальной замены переменных внутри этих дифференциалов:

$$
\begin{array}{ll}
A \rightarrow \frac{A}{\sigma}, & t \rightarrow \frac{t}{\sigma^{2}} \quad \text { для симметрических представлений, } \\
A \rightarrow A \sigma, \quad q \rightarrow q \sigma^{2} & \text { для антисимметрических представлений. }
\end{array}
$$

Данная замена оставляет соответственно (5.1) и (5.2) инвариантными, что делает четырежды градуированные гомологии однородными для всех двухнитевых торических и твистованных узлов в случае симметрических и антисимметрических представлений. Этот результат имеет место благодаря специфическим свойствам дифференциалов, использованных для разложения полиномов узлов в случае симметрических представлений, а также вырожденности коэффициентов разложения. Явные замены переменных представлены в п. 5.2 и 5.3.

- Рекуррентные соотношения для суперполиномов, обсуждавшиеся в п. 4.4, можно также естественно преобразовать в соотношения для четырежды градуированных гомологий. Введение четвертой градуировки в эти соотношения эквивалентно ее введению в дифференциалы полиномов узлов. Мы вкратце иллюстрируем это утверждение в п. 5.6.

Заметим, что мы уже давали конкретные комментарии такого рода в подходящих местах текста, теперь мы собираемся проделать это более систематически. В остальной части данного раздела мы объясняем, что́ мы имеем в виду, несколько более детально.

5.2. Узел-“восьмерка", представления [1] и [2]. Оба примера, представленных в разделе 4.2 работы [24], являются в действительности полиномами от трех переменных. Если взять суперполином в переменных Макдональда $(A, q, t)$, тогда, используя подстановку

$$
A=\alpha \sqrt{-t_{\mathrm{r}}^{3} t_{\mathrm{c}}}, \quad q=-\kappa t_{\mathrm{c}}, \quad t=\kappa t_{\mathrm{r}},
$$

мы получаем четырежды градуированный ответ в переменных $\left(\alpha, \kappa, t_{\mathrm{c}}, t_{\mathrm{r}}\right)^{6)}$. После подстановки

$$
\alpha=t_{\mathrm{r}}^{-2} A \sqrt{\frac{t}{q}}, \quad \kappa=t_{\mathrm{r}}^{-1} t, \quad t_{\mathrm{c}}=-t_{\mathrm{r}} \frac{q}{t}
$$

мы получаем

$$
\mathcal{P}_{[1]}^{4_{1}}\left(\alpha, \kappa, t_{\mathrm{c}}, t_{\mathrm{r}}\right)=P_{[1]}^{4_{1}}(A, q, t)=1+\{A q\}\left\{\frac{A}{t}\right\},
$$

т. е. переменная $t_{\mathrm{r}}$ выпадает из ответа. Как уже было замечено в работе [24], в этом случае ответ зависит только от произведения $t_{\mathrm{r}} t_{\mathrm{c}}$. Таким образом, мы имеем три, а не четыре независимые переменные. Но в общем случае устранение четвертой

${ }^{6)}$ На протяжении этого раздела мы обозначаем четыре переменные $\left(a, q, t_{\mathrm{c}}, t_{\mathrm{r}}\right)$, использованные в [24], как $\left(\alpha, \kappa, t_{\mathrm{c}}, t_{\mathrm{r}}\right)$, чтобы избежать путаницы с переменными $a$ и $q$, использованными в настоящей работе. 
переменной является более сложной процедурой, которая задается заменой переменных (5.5). В частности, после такой подстановки четвертая переменная $t_{\mathrm{r}}$ также выпадает из выражения

$$
\mathcal{P}_{[2]}^{4_{1}}\left(\alpha, \kappa, t_{\mathrm{c}}, t_{\mathrm{r}}\right)=P_{[2]}^{4_{1}}(A, q, t)=1+\left(q+q^{-1}\right)\left\{A q^{2}\right\}\left\{\frac{A}{t}\right\}+\left\{A q^{3}\right\}\left\{A q^{2}\right\}\left\{\frac{A q}{t}\right\}\left\{\frac{A}{t}\right\} .
$$

В данном случае это можно было также видеть с самого начала, поскольку ответ однороден - все члены имеют одно и то же значение

$$
2 \#(\alpha)+\#(\kappa)-\#\left(t_{\mathrm{c}}\right)-\#\left(t_{\mathrm{r}}\right)=\mathrm{const}=0 .
$$

5.3. Трилистник в представлениях [1], [2] и [11]. Во-первых, заметим, что все ответы для (анти)симметрических представлений, представленные в разделе 4.1 работы [24], однородны и, таким образом, четвертая градуировка может быть полностью из них исключена. Для представлений [1] и [2] мы имеем

$$
2 \#(\alpha)+\#(\kappa)-\#\left(t_{\mathrm{c}}\right)-\#\left(t_{\mathrm{r}}\right)=\mathrm{const}
$$

с точно тем же инвариантом (5.6), что и для узла-“восьмерки” (ненулевое значение константы отвечает несущественному общему множителю). В то же время для представления [11] имеется другой инвариант, который выглядит совершенно другим образом:

$$
4 \#(\alpha)+\#(\kappa)-5 \#\left(t_{\mathrm{c}}\right)+\#\left(t_{\mathrm{r}}\right)=\text { const. }
$$

Заслуживает упоминания, что это соотношение неприменимо к трилистнику в представлении [1]. Причиной является специальный выбор градуировки $\left(\alpha, \kappa, t_{\mathrm{c}}, t_{\mathrm{r}}\right)$, который существенно зависит от количества строк в диаграмме Юнга. Кроме того, выбор градуировки $\left(\alpha, \kappa, t_{\mathrm{c}}, t_{\mathrm{r}}\right)$ ставит формулы преобразования зеркальной симметрии в зависимость от диаграммы (см. детали в разделе 3.3 работы [24]). Мы предпочитаем использовать набор переменных, который делает описание зеркальной симметрии универсальным для всех диаграмм:

$$
\alpha=A \sqrt{\frac{t}{q}}, \quad \kappa=t \sigma^{-1 / l(R)}, \quad t_{\mathrm{c}}=-\frac{q}{t} \sigma^{1 / l(R)}, \quad t_{\mathrm{r}}=\sigma^{-1 / l(R)},
$$

где $l(R)$ - число строк в соответствующей диаграмме Юнга $R$.

Зависимость от диаграммы в (5.8) компенсирует соответствующую зависимость правил зеркальной симметрии для переменных $\left(\alpha, \kappa, t_{\mathrm{c}}, t_{\mathrm{r}}\right)$. В терминах переменных $(A, q, t, \sigma)$ зеркальное преобразование является простой заменой

$$
A \longleftrightarrow A, \quad q \longleftrightarrow-\frac{1}{t}, \quad \sigma \longleftrightarrow \frac{1}{\sigma}
$$

для всех узлов и представлений.

Возвращение к регулярной трижды градуированной гомологии (суперполиномам), таким образом, достигается при $\sigma=1$. В то же время $(A, q, t)-$ просто переменные Макдонадьда. Четвертая градуировка $\sigma$ здесь в точности совпадает с градуировкой $Q$ в работе [24] (см. формулы в разделе 1.5 и предпоследнюю формулу в разделе 2.4 в [24]). 
Если теперь переписать $Z$-разложение четырежды градуированных конструкций в терминах переменных (5.8), легко заметить, что четвертая сигма-градуировка может быть восстановлена полностью алгоритмически из подходящего разложения суперполиномов, а именно

$$
\begin{aligned}
\left(\frac{q}{t}\right) \mathcal{P}_{[1]}^{3_{1}}= & 1-\left(\frac{A^{2} q}{t}\right)\left\{\frac{A \sigma}{t}\right\}\left\{\frac{A q}{\sigma}\right\} \\
\left(\frac{q}{t}\right)^{2} \mathcal{P}_{[2]}^{3_{1}}= & 1-\left(\frac{A^{2} q}{t}\right)\left(\left\{\frac{A q^{3}}{\sigma}\right\}\left\{\frac{A \sigma}{t}\right\}+\left\{\frac{A q}{\sigma}\right\}\left\{\frac{A \sigma}{t}\right\}\right)+ \\
& +\left(\frac{A^{2} q}{t}\right)^{2} q^{2}\left\{\frac{A q^{3}}{\sigma}\right\}\left\{\frac{A q^{2}}{\sigma}\right\}\left\{\frac{A q \sigma}{t}\right\}\left\{\frac{A \sigma}{t}\right\}, \\
\left(\frac{q}{t}\right)^{2} \mathcal{P}_{[11]}^{3_{1}}= & 1-\left(\frac{A^{2} q}{t}\right)\left(\left\{\frac{A \sigma}{t^{3}}\right\}\left\{\frac{A q}{\sigma}\right\}+\left\{\frac{A \sigma}{t}\right\}\left\{\frac{A q}{\sigma}\right\}\right)+ \\
& +\left(\frac{A^{2} q}{t}\right)^{2} t^{-2}\left\{\frac{A \sigma}{t^{3}}\right\}\left\{\frac{A \sigma}{t^{2}}\right\}\left\{\frac{A q}{\sigma}\right\}\left\{\frac{A q}{t \sigma}\right\} .
\end{aligned}
$$

Строительные блоки $Z$-разложения - это пары, составленные из двух различных типов дифференциалов. Чтобы сделать описание более наглядным, можно ассоциировать один дифференциал с $x$-координатой конкретной клетки диаграммы Юнга, а другой - с $y$-координатой (однако это не подразумевает точного разделения, см. детальное описание в п. 3.6). Чтобы восстановить переменную $\sigma$, нужно масштабировать $A$ в дифференциалах первого типа, как $A \rightarrow A / \sigma$, а в дифференциалах второго типа подставить $A \rightarrow A \sigma$. Это же правило выполняется для описанных примеров узла-“восьмерки”, для трилистника в представлении [22] и для узла $T^{3,4}$ в представлениях [1], [2], т. е. для всех примеров из статьи [24]. Мы полагаем, что это будет общим правилом восстановления четырежды градуированной гомологии.

Наконец, нам надо сделать замечание о специфике симметрических и антисимметрических представлений. В терминах переменных $(A, q, t, \sigma)$, заданных формулами (5.8), формулы (5.7) превращаются в

$$
\begin{aligned}
& \#(A)+2 \#(t)+\#(\sigma)=\text { const } \\
& \#(A)-2 \#(q)-\#(\sigma)=\text { const. }
\end{aligned}
$$

Иными словами, если взять суперполином $P_{[r]}^{3_{1}}$ узла $3_{1}$ в симметрическом представлении и полином $P_{\left[1^{r}\right]}^{3_{1}}$ в антисимметрическом представлении, то четырежды градуированная гомология может быть восстановлена при помощи простой замены переменных:

$$
\begin{aligned}
\mathcal{P}_{[r]}^{3_{1}} & =P_{[r]}^{3_{1}}\left(\frac{A}{\sigma}, q, \frac{t}{\sigma^{2}}\right), \\
\mathcal{P}_{\left[1^{r}\right]}^{3_{1}} & =P_{\left[1^{r}\right]}^{3_{1}}\left(A \sigma, q \sigma^{2}, t\right) .
\end{aligned}
$$

Мы предполагаем, что это верно для всех двухнитевых торических и твистованных узлов во всех симметрических и антисимметрических представлениях. 
5.4. Трилистник в представлении [22]. Для представления [22] после исправления двух незначительных опечаток ответ из работы [24] замечательно разлагается в терминах мультидифференциалов. А именно, перепишем представленные полиномы в терминах переменных $(A, q, t, \sigma)$, заданных формулами $(5.8)$, для числа столбцов $R=2$ :

$$
\alpha=A \sqrt{\frac{t}{q}}, \quad \kappa=\frac{t}{\sqrt{\sigma}}, \quad t_{\mathrm{c}}=-\frac{q \sqrt{\sigma}}{t}, \quad t_{\mathrm{r}}=\frac{1}{\sqrt{\sigma}}
$$

(в этих терминах $\left.\gamma=t_{\mathrm{c}} t_{\mathrm{r}}=-q / t=T\right)$. Тогда мы имеем для полинома $\mathcal{P}_{[22]}^{3_{1}}$ из работы [24] формулу

$$
\begin{aligned}
\left(\frac{q}{t}\right)^{4} \mathcal{P}_{[22]}^{3_{1}}= & 1-A^{2}\left\{\frac{A q^{2}}{\sigma}\right\}\left\{\frac{A \sigma}{t^{2}}\right\} \times \\
& \times\left[-\left(\frac{q}{t}\right)^{8} A^{6}\left\{\frac{A q^{3}}{\sigma}\right\}\left\{\frac{A \sigma}{t^{3}}\right\}\left\{\frac{A q^{3}}{t \sigma}\right\}\left\{\frac{A q \sigma}{t^{3}}\right\}\left\{\frac{A q^{2}}{t \sigma}\right\}\left\{\frac{A q \sigma}{t^{2}}\right\}+\right. \\
& +A^{4}\left(\frac{q^{6}}{t^{4}}+\frac{q^{4}}{t^{4}}+\frac{q^{6}}{t^{6}}+\frac{q^{4}}{t^{6}}\right)\left\{\frac{A q^{3}}{\sigma}\right\}\left\{\frac{A \sigma}{t^{3}}\right\}\left\{\frac{A q^{2}}{t \sigma}\right\}\left\{\frac{A q \sigma}{t^{2}}\right\}- \\
& -\left(\frac{q}{t}\right)^{3} A^{2}\left(q t^{2}\left(t+t^{-1}\right)\left\{\frac{A q^{3}}{\sigma}\right\}\left\{\frac{A q \sigma}{t^{2}}\right\}+\frac{q+q^{-1}}{q^{2} t}\left\{\frac{A q^{2}}{t \sigma}\right\}\left\{\frac{A \sigma}{t^{3}}\right\}+\right. \\
& \left.\left.+\left\{\frac{A q^{2} t}{\sigma}\right\}\left\{\frac{A q \sigma}{t^{2}}\right\}+\left\{\frac{A q^{2}}{t \sigma}\right\}\left\{\frac{A \sigma}{q t^{2}}\right\}\right)+\frac{q}{t}\left(q+q^{-1}\right)\left(t+t^{-1}\right)\right] .
\end{aligned}
$$

5.5. Трехнитевые торические узлы $T^{3,4}$. Единственный пример трехнитевого торического узла в работе [24] - это $T^{3,4}$. Применяя замену переменных (5.8) к результатам, представленным в разделе 4.3 работы [24], заметим, что соотношения (5.9), (5.10) более неверны. Вместо этого четырежды градуированная конструкция разделяется на несколько однородных частей. В простейшем примере фундаментального представления имеются две части:

$$
\left(\frac{q}{t}\right)^{3} \mathcal{P}_{[1]}^{[3,4]}(A, q, t, \sigma)=\operatorname{Pr}_{0}\left(\mathcal{P}_{\square}^{[3,4]}\right)+\frac{q}{t} \operatorname{Pr}_{1}\left(\mathcal{P}_{\square}^{[3,4]}\right),
$$

где $\mathbf{P r}_{k}$ обозначает проектор на однородную часть степени $k$ (полном $P_{\square}^{[2,7]}$ здесь можно взять из п. 3.4, нам он нужен только как функция трех переменных безо всяких ссылок на его $Z$-разложение):

$$
\begin{aligned}
& \operatorname{Pr}_{0}\left(\mathcal{P}_{\square}^{[3,4]}\right)=\mathcal{P}_{\square}^{[2,7]}=P_{\square}^{[2,7]}\left(\frac{A}{\sigma}, q, \frac{t}{\sigma^{2}}\right), \\
& \operatorname{Pr}_{1}\left(\mathcal{P}_{\square}^{[3,4]}\right)=\frac{t}{q}\left(\mathcal{P}_{\square}^{[3,4]}-\mathcal{P}_{\square}^{[2,7]}\right)=\left(\frac{A^{2} q}{t}\right)^{4}\left\{\frac{A q}{\sigma}\right\}\left\{\frac{A \sigma}{t}\right\} .
\end{aligned}
$$

Однородность здесь понимается по отношению к масштабированию (5.3), что позволяет свести любую однородную часть к функции меньшего числа переменных, т. е. убрать четвертую градуировку $\sigma$. Напротив, в сумме нескольких членов с разными однородностями это масштабирование должно генерировать множители $\sigma$ в разных 
степенях. В частности, в этом конкретном случае причина, почему полином $\mathcal{P}_{[1]}^{[3,4]}$ не является однородным, заключается в присутствии дополнительного фактора $q / t$ в $Z$-разложении суперполинома. В отличие от комбинации $A^{2} q / t$, множитель $q / t$ не инвариантен относительно замены (5.12). Это говорит о том, что источником четвертой градуировки $\sigma$ является разное масштабирование двух типов дифференциалов, которые входят в $Z$-разложение парами. Опять же мы предполагаем, что переход к четырежды градуированной конструкции не влияет на коэффициенты разложения $g_{R, j}^{\mathcal{K}}$ для всех узлов $\mathcal{K}$ и представлений $R$.

Разложение (5.13) все еще не слишком впечатляет благодаря крайней простоте суперполинома торического узла $T^{3,4}$ в фундаментальном представлении, однако мы начали с этого тривиального примера, чтобы продемонстрировать общую концепцию. Наш подход становится более замечательным при его применении к четырежды градуированному полиному $\mathcal{P}_{[2]}^{3,4}$. Здесь мы имеем

$$
\left(\frac{q}{t}\right)^{6} \mathcal{P}_{[2]}^{[3,4]}=\operatorname{Pr}_{0}\left(\mathcal{P}_{[2]}^{[3,4]}\right)+\frac{q}{t} \operatorname{Pr}_{1}\left(\mathcal{P}_{[2]}^{[3,4]}\right)+\left(\frac{q}{t}\right)^{2} \operatorname{Pr}_{2}\left(\mathcal{P}_{[2]}^{[3,4]}\right),
$$

где

$$
\begin{aligned}
\operatorname{Pr}_{0}\left(\mathcal{P}_{[2]}^{[3,4]}\right) & =\operatorname{Pr}_{0}\left(P_{[2]}^{[3,4]}\right)\left(\frac{A}{\sigma}, q, \frac{t}{\sigma}\right)=P_{[2]}^{[2,7]}\left(\frac{A}{\sigma}, q, \frac{t}{\sigma^{2}}\right), \\
\operatorname{Pr}_{1}\left(\mathcal{P}_{[2]}^{[3,4]}\right) & =\operatorname{Pr}_{1}\left(P_{[2]}^{[3,4]}\right)\left(\frac{A}{\sigma}, q, \frac{t}{\sigma}\right), \\
\operatorname{Pr}_{2}\left(\mathcal{P}_{[2]}^{[3,4]}\right) & =\operatorname{Pr}_{2}\left(P_{[2]}^{[3,4]}\right)\left(\frac{A}{\sigma}, q, \frac{t}{\sigma}\right)= \\
& =\left(\frac{A^{2} q}{t}\right)^{8} q^{12}\left\{\frac{A q^{3}}{\sigma}\right\}\left\{\frac{A q^{2}}{\sigma}\right\}\left\{\frac{A q \sigma}{t}\right\}\left\{\frac{A \sigma}{t}\right\} .
\end{aligned}
$$

Соотношение (5.16) выглядит прямым обобщением (5.14). Что касается высшей степени однородности, то, если сравнить (5.18) с (5.15), естественно трактовать коэффициент разложения во втором симметрическом представлении как квадрат соответствующего коэффициента в фундаментальном представлении по отношению некоторой градуированной операции о', подобной операции из п. 3.3.1:

$$
a^{8} \circ^{\prime} a^{8}=q^{12} a^{16} .
$$

Наиболее интересная часть соотношений (5.16)-(5.18) - это опущенная левая часть равенства (5.17). Используя $a$ как естественную переменную в коэффициентах разложения, можно записать

$$
\begin{aligned}
\operatorname{Pr}_{1}\left(\mathcal{P}_{[2]}^{[3,4]}\right)= & {[2]_{q}\left\{\frac{A q^{2}}{\sigma}\right\}\left\{\frac{A \sigma}{t}\right\} \times } \\
& \times\left[a^{8}-\left\{\frac{A q^{3}}{\sigma}\right\}\left\{\frac{A q \sigma}{t}\right\}\left(\left(a^{18} q^{19}+a^{14} q^{10}[2]_{q}+a^{10} q[3]_{q}\right)-\right.\right. \\
& \left.\left.-\left\{\frac{A q^{4}}{\sigma}\right\}\left\{\frac{A q^{2} \sigma}{t}\right\}\left(a^{16} q^{15}[2]_{q}+a^{12} q^{4}[3]_{q}\right)-\left\{\frac{A q^{5}}{\sigma}\right\}\left\{\frac{A q^{3} \sigma}{t}\right\} a^{14} q^{9}\right)\right] .
\end{aligned}
$$


В терминах коэффициентов $g$ мы имеем

$$
g=[2]_{q}\left[0,-a^{8}, a^{18} q^{19}+a^{14} q^{10}[2]_{q}+a^{10} q[3]_{q},-a^{16} q^{15}[2]_{q}-a^{12} q^{4}[3]_{q}, a^{7} q^{9}\right] .
$$

Опять заметим, что во всех формулах (5.16)-(5.18) четвертая градуировка не влияет на коэффициенты $g$ разложения по базису мультидифференциалов.

5.6. Рекуррентные соотношения. Алгоритмический способ введения дополнительной градуировки позволяет непосредственно деформировать различные соотношения в четырежды градуированном случае. К примеру, согласно описанным правилам уравнения из п. 4.4 прямо обобщаются на случай четырежды градуированных полиномов. Так, для трилистника

$$
\begin{aligned}
\left(\frac{q}{t}\right)^{2} \mathcal{P}_{[22]}- & \mathcal{P}_{[11]}=\left\{\frac{A q^{3}}{\sigma}\right\}\left\{\frac{A \sigma}{t^{2}}\right\} \times \\
& \times\left(A^{14} q^{15} t^{-16}-A^{12} q^{15} \sigma^{-2} t^{-10}-A^{12} q^{13} \sigma^{-2} t^{-12}-A^{12} q^{13} \sigma^{-2} t^{10}-\right. \\
& -A^{12} q^{11} \sigma^{2} t^{-16}-A^{12} q^{11} \sigma^{2} t^{-14}-A^{12} q^{9} \sigma^{2} t^{-14}+ \\
& +A^{10} q^{13} \sigma^{-4} t^{-6}+A^{10} q^{13} \sigma^{-4} t^{-4}+A^{10} q^{11} t^{-10}+A^{10} q^{11} t^{-8}+ \\
& +A^{10} q^{11} \sigma^{-4} t^{-6}+A^{10} q^{9} t^{-12}+A^{10} q^{9} t^{-10}+A^{10} q^{9} t^{-8}+ \\
& +A^{10} q^{7} \sigma^{4} t^{-14}+A^{10} q^{5} \sigma^{4} t^{-14}+A^{10} q^{5} \sigma^{4} t^{-12}-A^{8} q^{11} \sigma^{-6}- \\
& -A^{8} q^{9} \sigma^{-2} t^{-6}-A^{8} q^{9} \sigma^{-2} t^{-4}-A^{8} q^{7} \sigma^{2} t^{-8}+A^{8} q^{5} \sigma^{-2} t^{-6}- \\
& \left.-A^{8} q \sigma^{6} t^{-12}-A^{6} q^{5} \sigma^{-4}-A^{6} q^{3} t^{-6}-A^{6} q^{3} t^{-4}-A^{6} q \sigma^{4} t^{-8}\right),
\end{aligned}
$$

и деформация дифференциалов в правой части такая, как ожидалось.

\section{6. ЗАКЛЮЧЕНИЕ}

При изучении любой конкретной модели квантовой теории поля или теории струн критически важным является понимание того, что́ является подходящим базисом для ее корреляционных функций. В теории Черна-Саймонса по крайней мере два таких базиса надежно идентифицированы - это базис хордовых диаграмм, играющий важную в теории инвариантов Васильева [31] и разложении по родам [32], и базис характеров группы $S U(\infty)$ (функции Шура и Макдональда), естественно возникающий [11], [33], [34] при реализации узлов в виде кос и позволяющий ввести (расширенные) полиномы узлов вне массовой оболочки в духе работы [12].

В настоящей работе мы утверждаем, что базис, обеспеченный $Z$-разложением [20], может быть не менее важным, а для некоторых целей даже более хорошим, чем разложение по характерам. Имеется целый ряд аргументов в пользу этого исследования.

- История начинается со свойства факторизации (2.4) специальных полиномов (т. е. при $q=t=1$ ), и первой целью является обобщить это свойство на случай полиномов ХОМФЛИ и суперполиномов настолько непосредственно, насколько это возможно. Важным признаком того, что это хорошо мотивированная задача, было частное (только по одной переменной), но буквальное обобщение, по крайней мере на конкретные представления, в работе [29]. 
- Второе критическое наблюдение состоит в том, что специальные полиномы, будучи функциями только от $A$, в действительности естественно разлагаются по степеням самой переменной $A$ и переменной $\{A\}^{2}=(A-1 / A)^{2}$. Это звучит странно, и, конечно, такое биразложение не определено полностью на уровне специальных полиномов. Зато оно сохраняет некоторую нетривиальную информацию о структуре общих раскрашенных суперполиномов. Если взять $t \neq 1$, то можно было бы положить $A=t^{N}$ и рассмотреть разложение по степеням параметра $\bar{\hbar}=\ln t$, где $\{A\}$ имело бы порядок $\bar{\hbar}$. При этом разложение по $\{A\}$ - это остаток такого разложения, и степени $A$ вводятся так, что новые степени $\bar{\hbar}$ не добавляются. Эта информация о скрытой структуре полиномов узлов наглядно обобщается на следующем шаге нашего рассуждения.

- Следующее наблюдение состоит просто в том, что каждая переменная $\{A\}$ есть предел $q=t=1$ некоторого дифференциала ДГР $D_{j}^{i}=\left\{A q^{i} / t^{j}\right\}$. Таким образом, разложение по $\{A\}$ с зависящими от $A$ коэффициентами специальных полиномов приходит из соответствующего разложения полного суперполинома. Для простейших (анти)симметрических представлений это разложение имеет специальный вид

$$
P_{[r]}=1+Z_{1 \mid 1}\left(g_{1}+Z_{2 \mid 1}\left(g_{2}+Z_{3 \mid 1}\left(g_{3}+\cdots\right)\right)\right),
$$

где $Z_{i \mid 1}=\left\{A q^{i}\right\}\{A / t\}$, отсюда два термина $-Z$-разложение и иерархия дифференциалов. В общем случае это разложение действительно описывает произвольный раскрашенный суперполином в терминах коэффициентов $g_{R}[I]$ его разложения по мультидифференциалам.

- Критическим свойством разложения является тот факт, что набор коэффициентов $g_{R}[I](A, q, t)$ много проще, чем кажется. Это выглядит так, что если мы знаем $g_{\square}(A, q, t)$ при $q=t$ для $R=\square$ и для полиномов ХОМФЛИ, то этого может быть, в конце концов, достаточно, чтобы найти все $g_{R}^{[I]}$ как определенные степени коэффициента $g_{\square}$ по отношению к некоторым зависящим от $R$ неассоциативным умножениям и коумножениям и с алгоритмически определенной $t$-деформацией. Более того, похоже, что другие градуировки, включая градуировку, предложенную в работе [24], можно также алгоритмически ввести, если $Z$-разложение известно.

В настоящей статье мы привели только очень ограниченный набор аргументов в поддержку этих наблюдений. Иерархия дифференциалов весьма трудоемка даже в простейших примерах. Более того, она не всегда сводится к $Z$-факторам: как известно из примера представления [21] (см. статью [23]), могут возникать некоторые $\epsilon^{2}$-члены даже с нечетным количеством дифференциалов, которые нужно соответствующим образом понять и учесть. Тем не менее мы верим, что эти указания достаточно убедительно оправдывают необходимость изучения иерархии дифференциалов наряду с другими общими подходами к полиномам узлов.

Благодарности. Наша работа частично поддержана Министерством образования и науки РФ (контракт 8498), Brazil National Counsel of Scientific and Technological Development (А. Mop.), Программой поддержки ведущих научных школ (грант НШ-3349.2012.2 (С. А.)), РФФИ (гранты № 12-02-00594 (С. А.), 13-02-00457 (А. Мир.) и 13-02-00478 (А. Мор.), а также гранты № 12-01-33071 мол_а_вед (С.А.), совместные гранты 12-02-92108-ЯФ_a, 13-02-91371-CT_a). 


\section{Список литературы}

[1] J. W. Alexander, Trans. Amer. Math. Soc., 30:2 (1928), 275-306; J. H. Conway, "An enumeration of knots and links, and some of their algebraic properties", Computational Problems in Abstract Algebra (Oxford, 29 August-2 September, 1967), ed. J. Leech, Pergamon, Oxford, 1970, 329-358; V.F. R. Jones, Invent. Math., 72:1 (1983), 1-25; Bull. Amer. Math. Soc. (N.S.), 12:1 (1985), 103-111; Ann. Math. (2), 126:2 (1987), 335-388; L. Kauffman, Topology, 26:3 (1987), 395-407; P. Freyd, D. Yetter, J. Hoste, W. B. R. Lickorish, K. Millet, A. Ocneanu, Bull. Amer. Math. Soc. (N.S.), 12:2 (1985), 239-246; J. H. Przytycki, K. P. Traczyk, Kobe J. Math., 4:2 (1987), 115-139.

[2] S.-S. Chern, J. Simons, Ann. Math. (2), 99:1 (1974), 48-69.

[3] A. S. Schwarz, "New topological invariants arising in the theory of quantized fields", International Conference on Topology, Abstracts (Part II) (Baku, 1987), Math. Institute, Aserbaijan Acad. Sci., Baku, 1987; E. Witten, Commun. Math. Phys., 121:3 (1989), 351-399.

[4] R. Gopakumar, C. Vafa, Adv. Theor. Math. Phys., 3:5 (1999), 1415-1443, arXiv: hep-th/9811131; H. Ooguri, C. Vafa, Nucl. Phys. B, 577:3 (2000), 419-438, arXiv: hep-th/9912123; J. Labastida, M. Mariño, Commun. Math. Phys., 217:2 (2001), 423-449, arXiv: hep-th/0004196; M. Mariño, C. Vafa, Framed knots at large $N$, arXiv: hep-th/0108064; S. Gukov, A. Schwarz, C. Vafa, Lett. Math. Phys., 74:1 (2005), 53-74, arXiv: hep-th/0412243.

[5] M. Khovanov, Duke Math. J., 101:3 (2000), 359-426, arXiv: math/9908171; Exp. Math., 12:3 (2003), 365-374, arXiv: math/0201306; J. Knot Theory Ramifications, 14:1 (2005), 111-130, arXiv: math/0302060; Algebr. Geom. Topol., 4 (2004), 1045-1081, arXiv: math/0304375.

[6] M. Khovanov, L. Rozhansky, Fund. Math., 199:1 (2008), 1-91, arXiv: math.QA/0401268; Geom. Topol., 12:3 (2008), 1387-1425, arXiv: math.QA/0505056; D. Bar-Natan, Algebr. Geom. Topol., 2 (2002), 337-370, arXiv: math/0201043.

[7] V. Dolotin, A. Morozov, JHEP, 01 (2013), 065, 46 pp., arXiv: 1208.4994; J. Phys.: Conf. Ser., 411:1 (2013), 012013, 22 pp., arXiv: 1209.5109.

[8] N. M. Dunfield, S. Gukov, J. Rasmussen, Exp. Math., 15 (2006), 129-159, arXiv: math/0505662.

[9] H. Itoyama, A. Mironov, A. Morozov, And. Morozov, Internat. J. Modern Phys. A, 27:19 (2012), 1250099, 85 pp., arXiv: 1204.4785; 28:3-4 (2013), 1340009, 81 pp.; A. Anokhina, A. Mironov, A. Morozov, And. Morozov, Nucl. Phys. B, 868 (2013), 271-313, arXiv: 1207.0279; A. Anokhina, A. Mironov, A. Morozov, And. Morozov, Adv. High Enegry Phys., 13 (2013), 931830, 12 pp., arXiv: 1304.1486.

[10] E. Guadagnini, M. Martellini, M. Mintchev, "Chern-Simons field theory and quantum groups", Quantum groups, Proceedings of the 8th International Workshop on Mathematical Physics (Arnold Sommerfeld Institute, Clausthal, Germany, 19-26 July, 1989), Lecture Notes in Physics, 370, eds. H.-D. Doebner, J.-D. Hennig, Springer, Berlin, 307-317; Phys. Lett. B, 235:3-4 (1990), 275-281; N. Yu. Reshetikhin, V. G. Turaev, Commun. Math. Phys., 127:1 (1990), 1-26; A. Morozov, A. Smirnov, Nucl. Phys. B, 835:3 (2010), 284-313, arXiv: 1001.2003.

[11] P. Dunin-Barkowski, A. Mironov, A. Morozov, A. Sleptsov, A. Smirnov, JHEP, 03 (2013), 021, 85 pp., arXiv: 1106.4305.

[12] A. Mironov, A. Morozov, And. Morozov, "Character expansion for HOMFLY polynomials. I. Integrability and difference equations", Strings, Gauge Fields, and the Geometry Behind: The Legacy of Maximilian Kreuzer, eds. A. Rebhan, L. Katzarkov, J. Knapp, R. Rashkov, E. Scheidegger, World Sci., Singapore, 2013, 101-118, arXiv: 1112.5754.

[13] M. Aganagic, Sh. Shakirov, Knot homology from refined Chern-Simons theory, arXiv: 1105.5117; Refined Chern-Simons theory and topological string, arXiv: 1210.2733.

[14] I. Cherednik, Jones polynomials of torus knots via DAHA, arXiv: 1111.6195. 
[15] N. Carqueville, D. Murfet, Algebr. Geom. Topol., 14 (2014), 489-537, arXiv: 1108.1081; A. Oblomkov, J. Rasmussen, V. Shende, The Hilbert scheme of a plane curve singularity and the HOMFLY homology of its link, arXiv: 1201.2115; E. Gorsky, A. Oblomkov, J. Rasmussen, V. Shende, Torus knots and the rational DAHA, arXiv: 1207.4523.

[16] S. Gukov, M. Stosic, Homological algebra of knots and BPS states, arXiv: 1112.0030.

[17] A. Mironov, A. Morozov, And. Morozov, "Evolution method and "differential hierarchy" of colored knot polynomials", Nonlinear and modern mathematical physics, Proceedings of the 2nd International Workshop (Tampa, Florida, USA, 9-11 March, 2013), AIP Conference Proceedings, 1562, eds. W.-X. Ma, D. Kaup, AIP, Melville, NY, 2013, 123-155, arXiv: 1306.3197.

[18] E. Gorsky, "q,t-Catalan numbers and knot homology", Zeta Functions in Algebra and Geometry, Contemporary Mathematics, 566, ed. A. Campillo, AMS, Providence, RI, 2012, 213-232, arXiv: 1003.0916.

[19] A. Negut, Moduli of flags of sheaves on $P^{2}$ and their K-theory, arXiv: 1209.4242; E. Gorsky, A. Negut, Refined knot invariants and Hilbert schemes, arXiv: 1304.3328.

[20] H. Itoyama, A. Mironov, A. Morozov, And. Morozov, JHEP, 07 (2012), 131, 21 pp., arXiv: 1203.5978.

[21] S. Nawata, P. Ramadevi, Zodinmawia, X. Sun, JHEP, 11 (2012), 157, 38 pp., arXiv: 1209.1409; H. Fuji, S. Gukov, M. Stosic, P. Sulkowski, 3d analogs of Argyres-Douglas theories and knot homologies, arXiv: 1209.1416.

[22] H. Fuji, S. Gukov, P. Sulkowski, Volume conjecture: refined and categorified, arXiv: 1203.2182 .

[23] A. Anokhina, A. Mironov, A. Morozov, And. Morozov, Knot polynomials in the first non-symmetric representation, arXiv: 1211.6375.

[24] E. Gorsky, S. Gukov, M. Stosic, Quadruply-graded colored homology of knots, arXiv: 1304.3481.

[25] A. Mironov, A. Morozov, And. Morozov, JHEP, 03 (2012), 034, 34 pp., arXiv: 1112.2654.

[26] R. Gelca, Math. Proc. Cambridge Philos. Soc., 133:2 (2002), 311-323, arXiv: math/0004158; R. Gelca, J. Sain, J. Knot Theory Ramifications, 12:2 (2003), 187-201, arXiv: math/0201100; S. Gukov, Commun. Math. Phys., 255:3 (2005), 577-627, arXiv: hep-th/0306165; S. Garoufalidis, "On the characteristic and deformation varieties of a knot", Proceedings of the Casson Fest (Fayetteville, AR, USA, April 10-12, 2003), Geometry and Topology Monographs, 7, eds. C. Gordon, Y. Rieck, Geom. Topol. Publ., Coventry, 2004, 291-309, arXiv: math/0306230; H. Fuji, S. Gukov, P. Sułkowski, Super-A-polynomial for knots and BPS states, arXiv: 1205.1515.

[27] A. Mironov, A. Morozov, "Equations on knot polynomials and 3d/5d duality", The Sixth International School on Field Theory and Gravitation-2012 (Petrópolis, RJ, Brazil 23-27 April, 2012), AIP Conference Proceedings, 1483, eds. W. A. Rodrigues, Jr., R. Kerner, G. O. Pires, C. Pinheiro, AIP, Melville, NY, 2012, 189-211, arXiv: 1208.2282.

[28] M. Rosso, V. Jones, J. Knot Theory Ramifications, 2:1 (1993), 97-112; X.-S. Lin, H. Zheng, Trans. Amer. Math. Soc., 362:1 (2010), 1-18, arXiv: math/0601267; S. Stevan, Ann. Henri Poincaré, 11 (2010), 1201-1224, arXiv: 1003.2861; A. Brini, B. Eynard, M. Mariño, Ann. Henri Poincaré, 13:8 (2012), 1873-1910, arXiv: 1105.2012.

[29] A. Morozov, JHEP, 12 (2012), 116, 7 pp., arXiv: 1208.3544; The first-order deviation of superpolynomial in an arbitrary representation from the special polynomial, arXiv: 1211.4596; Писвма в ЖКЭТФ, 97:3-4 (2013), 195-196.

[30] А. С. Анохина, А. А. Морозов, ТМФ, 178:1 (2014), 3-68.

[31] M. Kontsevich, "Vassiliev's Knot Invariants", I. M. Gel'fand Seminar, Advances in Soviet Mathematics, 16, Part 2, eds. S. I. Gel'fand, S. G. Gindikin, AMS, Providence, RI, 1993, 137-150; M. Alvarez, J. M. F. Labastida, E. Perez, Nucl. Phys. B, 488:3 (1997), 677-718, arXiv: hep-th/9607030; S. Chmutov, S. Duzhin, J. Mostovoy, Introduction to Vassiliev Knot Invariants, Cambridge Univ. Press, Cambridge, 2012, arXiv: 1103.5628. 
[32] А. Д. Миронов, А. Ю. Морозов, А.В. Слепцов, ТМФ, 177:2 (2013), 179-221, arXiv: 1303.1015; A. Mironov, A. Morozov, A. Sleptsov, Eur. Phys. J. C, 73:7 (2013), 2492, 7 pp., arXiv: 1304.7499; A. Morozov, "Integrability in non-perturbative QFT", Nonlinear and Modern Mathematical Physics, Proceedings of the 2nd International Workshop (Tampa, Florida, USA, 9-11 March, 2013), AIP Conference Proceedings, 1562, eds. W.-X. Ma, D. Kaup, AIP, Melville, NY, 2013, 167-176, arXiv: 1303.2578.

[33] A. Mironov, A. Morozov, Sh. Shakirov, A. Sleptsov, JHEP, 12 (2012), 70, 12 pp., arXiv: 1201.3339 .

[34] A. Mironov, A. Morozov, Sh. Shakirov, J. Phys. A, 45:35 (2012), 355202, 11 pp., arXiv: 1203.0667.

Поступила в редакцию 11.12.2013 